\title{
Experimental investigation of Mach- and Reynolds-number dependencies of the stall behavior of 2-element and 3- element high-lift wing sections
}

\author{
J. Wild ${ }^{1}$ \\ DLR German Aerospace Center, Braunschweig, 38108, Germany
}

\begin{abstract}
Experimental investigations have been carried out with the two-dimensional DLR-F15 high-lift wing section model in DNW-KKK cryogenic wind tunnel in order to differentiate between the influence of Mach- and Reynolds number on the stall behavior. Due to the cryogenic environment Mach- and Reynolds number have been varied independently between $M=0.1-0.25$ and $R e=1.4 \times 10^{6}-15.6 \times 10^{6}$. The investigation covers 2-element and 3-element configurations at various slat and flap settings and two different slat shapes. Focus of the investigation is to identify conditions of turbulent leading edge stall, shock related lift limitations and flap separations and their influence on achievable maximum lift coefficient.
\end{abstract}

$\begin{array}{ll}c & =\text { chord } \\ C_{p} & =\text { pressure coefficient } \\ C_{D} & =\text { drag coefficient } \\ C_{L} & =\text { lift coefficient } \\ \mathrm{d} t & =\text { time step } \\ M & =\text { Mach number } \\ \operatorname{Re} & =\text { Reynolds number } \\ T & =\text { static temperature } \\ \alpha & =\text { angle of attack }\end{array}$

\section{Nomenclature}

\section{Introduction}

$\mathrm{H}$

igh-lift system research is one major area of interest for today's civil transport aircraft development. Improvements in other fields like aero-acoustics or laminar wing are worthless if no suitable high-lift system can be identified. On the other hand these systems impose a severe weight penalty, asking for more optimized solutions designed to the edge of aerodynamic performance.

Current design relies more and more on computational methods, mainly RANS CFD. Unfortunately, for CFD validation purposes only few multi-element airfoils are available in the public, namely the British $\mathrm{L} 1 \mathrm{~T} 2{ }^{1}$, the Boeing TR-1332 $2^{2}$, and the McDonnell-Douglas 30P30N ${ }^{3,4}$ airfoils. All of them originate back into the 80's and 90's and data sets are only available for a fixed geometry. Design validation additionally requires the assessment of proper simulation of the sensitivities on changes of geometry and/or flow conditions.

Wind tunnel tests performed in ambient atmospheric low speed wind tunnels suffer the simultaneous dependency of Reynolds and Mach number on the flow velocity. Often Reynolds number effects are guessed by increasing the velocity, but for high-lift systems in the range of high angles of attack compressibility effects can get significant with local Mach numbers approaching or even overrunning Mach one. In this regime, Mach number and Reynolds number effects cannot be separated anymore, sometimes attributing compressibility effects to viscous reasons or vice versa.

In 2005 DLR started to set up a new 2D high-lift model for multiple purposes in high-lift research. The so-called DLR-F15 model was used up to now for investigations for wind-tunnel side-effects and corrections, noise reduced high-lift systems, flow separation control, sensitivities on geometrics, and the sensitivity on flow conditions reported here. Up to now the model has been used within 13 distinct test campaigns in the DNW-KKK and DNW-NWB wind

\footnotetext{
${ }^{1}$ Research Scientist, Institute of Aerodynamics and Flow Technology, Lilienthalplatz 7. AIAA Senior Member 1

American Institute of Aeronautics and Astronautics
} 
tunnels. Latest modifications include the implementation of advanced flow control techniques for use in the cryogenic environment and a setup as a finite span swept constant chord model in the DNW-NWB tunnel.

The main aim of the current investigation is to clearly distinct between Reynolds and Mach number sensitivities for a series of high-lift systems. The reported configurations include classical 3-element airfoils, 2-element airfoils without slat, and a noise-reducing slat configuration, and a target shape for a morphing leading. Special emphasis is put on the effects on the stall behavior.

\section{Experimental setup}

\section{A. Wind tunnel model DLR-F15}

The DLR-F15 wind tunnel model shown in Figure $\mathbf{1}$ is a 2D wall-to-wall high-lift model. The modular main wing allows exchanging leading and trailing edges. Therefore, different types of high-lift elements can be investigated and compared at the same baseline geometry. The clean wing section is derived from a generic high-lift wing investigated in the national funded project ProHMS ${ }^{5}$ and represents a state of the art transonic turbulent airfoil for a modern civil transport aircraft. A 3-element high-lift system comparable to state-of-the-art high-lift design is implemented as a reference. Beneath this, the model features a 2-element configuration with a clean leading edge or can be equipped with different kind of alternative leading and trailing edge devices. The devices are mounted on continuously adjustable brackets, allowing the free positioning of each element in all three degrees of freedom.

The model is equipped with about 220 static pressure probes. One dense pressure distribution is located in the center section and is used for the integration of the aerodynamic coefficients. In addition, two less dense pressure distributions are located closed to the tunnel walls in order to assess the two-dimensionality of the flow. Although, it has been discovered that the pressure distribution is not dense enough for an accurate integration of drag coefficients, leading to errors of up to $20 \%$. The pure integration error for lift coefficients is about $1 \%$ and for the pitching moment coefficient an accuracy of about $3 \%$ is achieved.

\section{A. Cryogenic wind tunnel DNW-KKK}
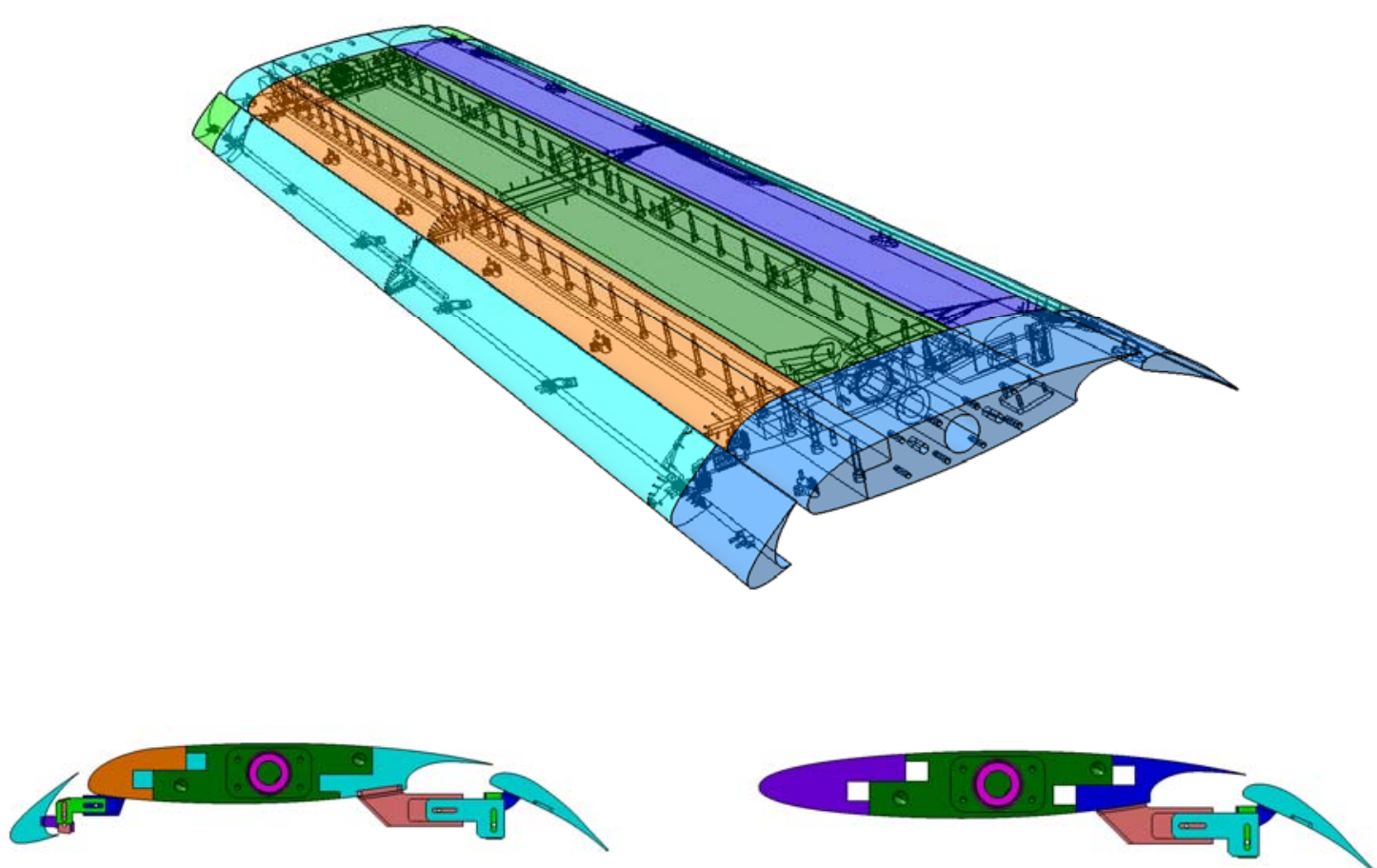

Figure 1. CAD assembly of the DLR-F15 2D high-lift model with 3-element and 2-element layout.

2

American Institute of Aeronautics and Astronautics 


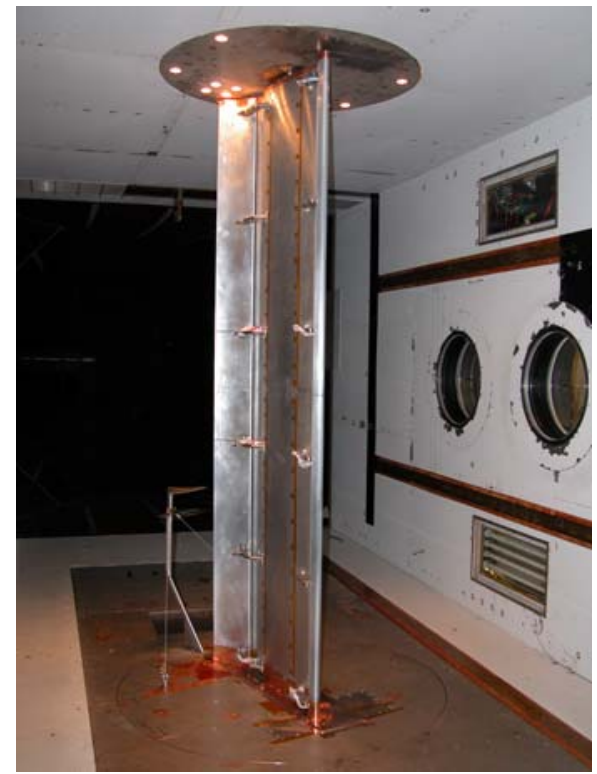

Figure 2. DLR-F15 model mounted in DNW-KKK test section.
The reported tests were carried out in the cryogenic facility DNWKKK in Cologne (Figure 2). The DNW-KKK is a closed loop lowspeed wind tunnel. It operates at approximately ambient pressure. Temperature can be regulated between ambient and $\mathrm{T}=100 \mathrm{~K}$. Mach number can range between $M=0.1$ and $M=0.3$. The test section has a cross section of $2.4 \times 2.4 \mathrm{~m}^{2}$. Based on the aerodynamic clean chord $c=0.6 \mathrm{~m}$ the maximum Reynolds number achieved was $R e=15.7 \times 10^{6}$. Figure 3 shows a sketch of the tunnel circuit including the system to inject liquid and gaseous nitrogen to control the tunnel temperature. The tunnel has a model conditioning room below the test section, which facilitates model adaptations while the tunnel can be kept in cold and cryogenic conditions. Nevertheless the usual time for a model modification takes about 6-8 hours including heating up and cooling down the model.

\section{B. Measurement techniques}

Beside the already mentioned static pressure probes two types of rakes were used in the tests. A total pressure wake rake with 91 pressure probes was mounted on one of the side turn-tables just after the flap trailing edge to inspect the total pressure losses (Figure 4). Earlier tests revealed that the use of a wake rake is erroneous for the evaluation of the drag coefficient of such a large high-lift model, especially at high angles of attack. To capture the wake completely the rake has to be positioned too close to the trailing edge. At high angles of attack the downwash of the model induces a cross flow with a deviation of the flow direction in the order of $30^{\circ}$ out of the rake axis, which is clearly out of the calibration range. At a more downstream position the wake is not captured completely and can hit the wall boundary layer, which again gives no accurate results. By the close up mounted rake on the turntable the axis of the rake is always aligned with the local flow direction. Nevertheless, the values measured correspond to the deficit in total pressure only and cannot directly be interpreted as drag coefficients. In order to assess the flow directionality and the 3-dimensionality of the flow, additionally for a few measurements a 5-hole-probe rake mounted on a traversing system was used. It consists of 15

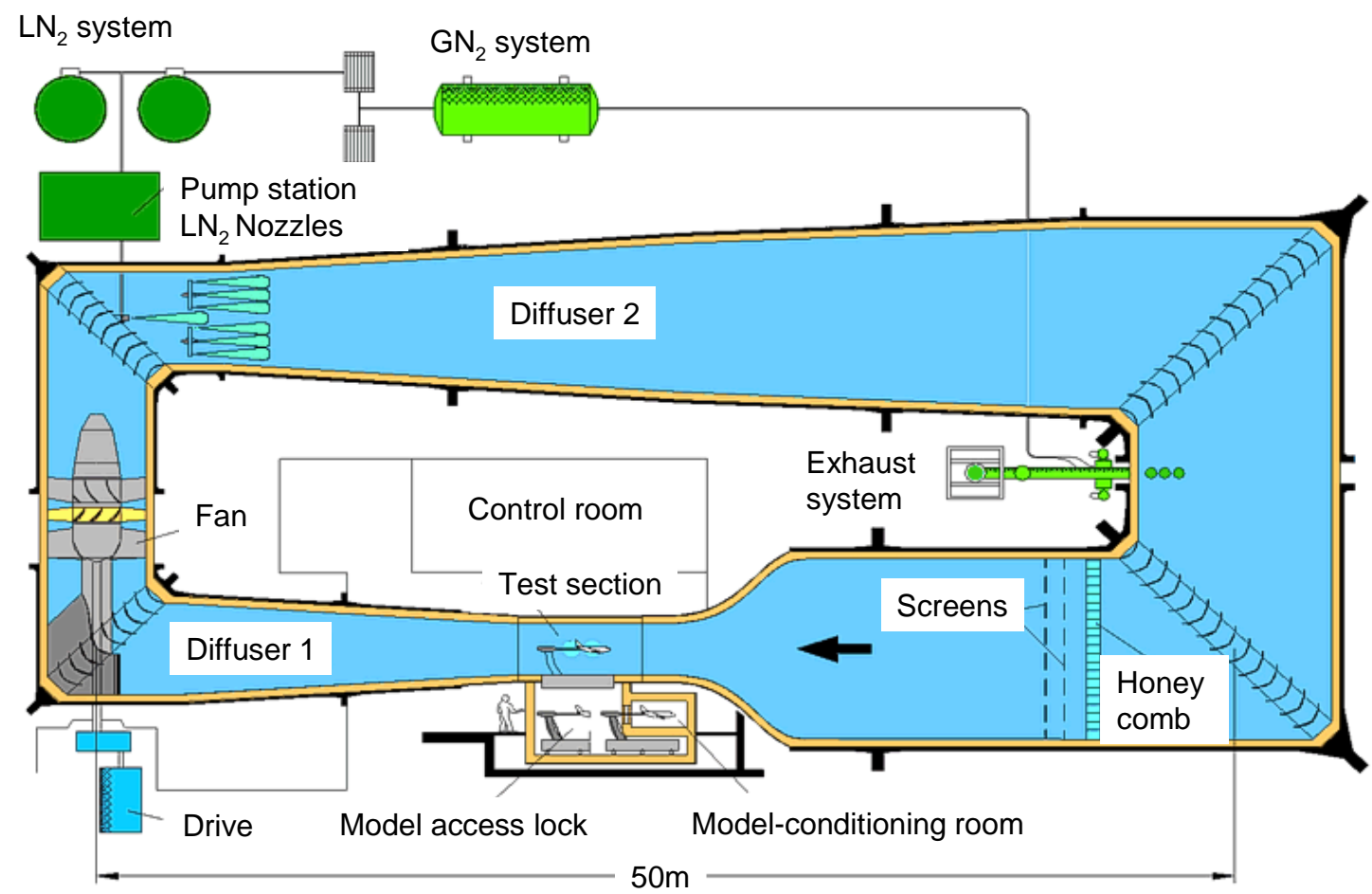

Figure 3. Sketch of DNW-KKK tunnel circuit.

3

American Institute of Aeronautics and Astronautics 
5-hole probes with a spacing of $25 \mathrm{~mm}$ between the axes.

Aerodynamic coefficients are obtained by integration of the pressure distribution only. Due to the integration error, especially for the drag coefficient, usual wall corrections are not applied. For the pressure coefficients a correction of the static pressure was applied based on the calibration of the tunnel.

\section{Test conditions}

Figure 5 shows the tunnel conditions used in the measurements. Mach- and Reynolds numbers were selected in order to independently assess Mach number and Reynolds number effects. The major design condition of the airfoil on aircraft level is $M=0.15$ at $R e=7 \times 10^{6}$. In the tests Reynolds number effects were assessed at three Mach numbers $M=[0.13,0.15,0.2]$ and Mach-number dependencies were assessed at two different Reynolds numbers $R e=\left[4 \times 10^{6}, 7 \times 10^{6}\right]$, although it has to be mentioned that with the current procedure the target Reynolds number spreads by about $5 \%$ from the target value. Additional measurements were taken at further points of interest, especially for the lowest Temperature at $T=100 \mathrm{~K}$ and the highest Mach number $\mathrm{M}=0.25$. Nevertheless, due to time and budget constraints it was not possible to measure all configurations for the full matrix of test conditions. Therefore, some of the comparisons made later on may

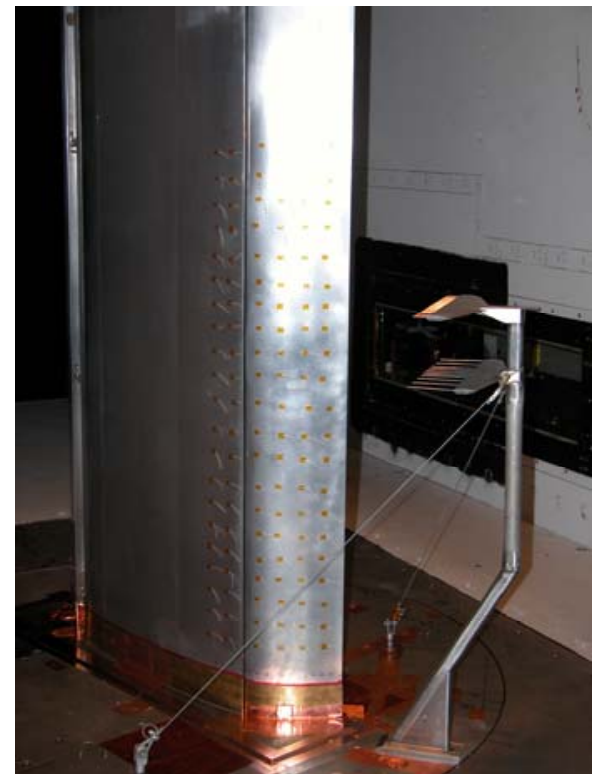

Figure 4. Wake rake mounted on turn table in DNW-KKK test section. miss some completeness in this sense.

\section{Test procedure and data analysis}

The experiments reported here were conducted within three separate test campaigns between 2006 and 2008. Of course not every configuration was investigated during each campaign. Proper comparisons should only be made for measurements obtained in the same campaign as the results of the long term repeatability later on reveals. Differences in the measured data of aerodynamic coefficients are attributed to a slightly different set of pressure probes available due to blocked ports on the model and for uncertainties of angle-of-attack adjustments.

The data always was obtained by full polar runs from negative angles of attack up beyond stall. The angle of attack was adjusted in a continuous mode with an angular velocity of $\mathrm{d} \alpha / \mathrm{d} t=0.2 \%$. Within the very first measurements this was compared to a step-by-step measurement and revealed no significant differences. The continuous mode offers a shorter run-time for one polar. Data points during the polar run have been taken even every quarter degree or every half degree, depending on the campaign.

For the measurements in cryogenic environment, the injection of nitrogen to control tunnel temperature was switched off during the acquisition of data for one polar. Previous experience has shown that switching on the temperature control leads to unpredicted disturbances that correlate directly with the temperature change. This

DLR-F15

Measurement DNW-KKK Envelope

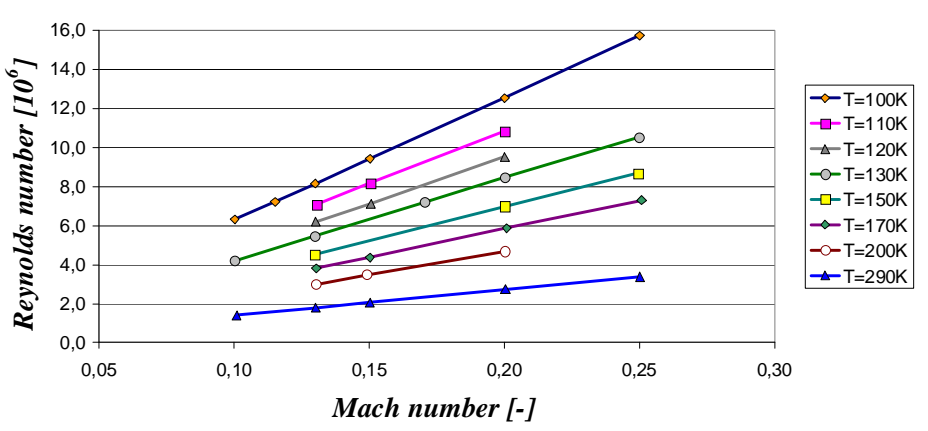
phenomenon is as more severe as the tunnel temperature goes towards its minimum, mainly due to the more significant influence on the actual Reynolds number. Within all the measurements reported here the tunnel was conditioned towards a temperature slightly below the designated value and during the polar the tunnel silently heated up slightly to a value slightly above it. The temperature drift reduces during the duration of the campaign mainly due to an increasing buffering effect of the tunnel insulation. It was decided that the temperature drift with a constant slope is beneficial over the occurrence of unpredicted temperature changes, especially when regarding polar

Figure 5. Test conditions during measurments of DLR-F15 plots. model in DNW-KKK. 


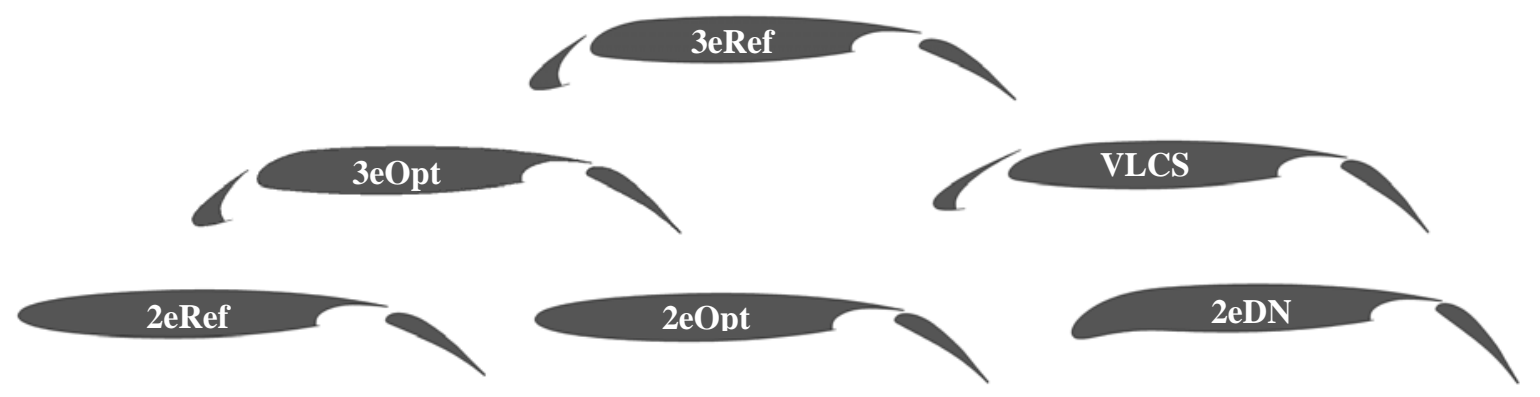

Figure 6. variations of the high-lift system of the DLR-F15 model

The data analysis reported in the follow on aims mainly on the stall behavior. In this sense the focus is on the evolution of maximum lift coefficient and corresponding angles of attack.

\section{Configurations}

Figure 6 gives an overview and introduces the nomenclature of the different configurations investigated. The baseline configuration is a 3-element configuration named F15-3eRef with a slat deployed at $28^{\circ}$ and a flap deployed at $35^{\circ}$. This wing section is directly taken from the reference 3D wing design and represents the landing configuration. Numerical optimization on this wing section has been used to identify a better aerodynamic design for the wing section only, while for the 3D wing also the span-wise effects of gap and overlap variation due to taper and wing twist has limited the design space. This configuration is denoted F15-3eOpt and has a higher deflection angle of the flap together with a reduced gap to keep the flap flow attached at low angles of attack combined with a comparable maximum lift coefficient. The third 3-element configuration shown here was designed in the scope of the DLR project LEISA ${ }^{6}$ for noise reduced high-lift systems, featuring a slat of significantly increased chord length, called F15-VLCS (very long chord slat).

The baseline for all 2-element configurations F15-2eRef is retrieved by retracting the slat of the F15-3eRef. Here again numerical optimization based on CFD was used to reposition the flap to get the optimum aerodynamic performance in absence of the slat device, resulting in configuration F15-2eOpt. Within the above mentioned project LEISA the aerodynamic shape of a smart morphing droop-nose was designed and incorporated into the model as F15-2eDN.
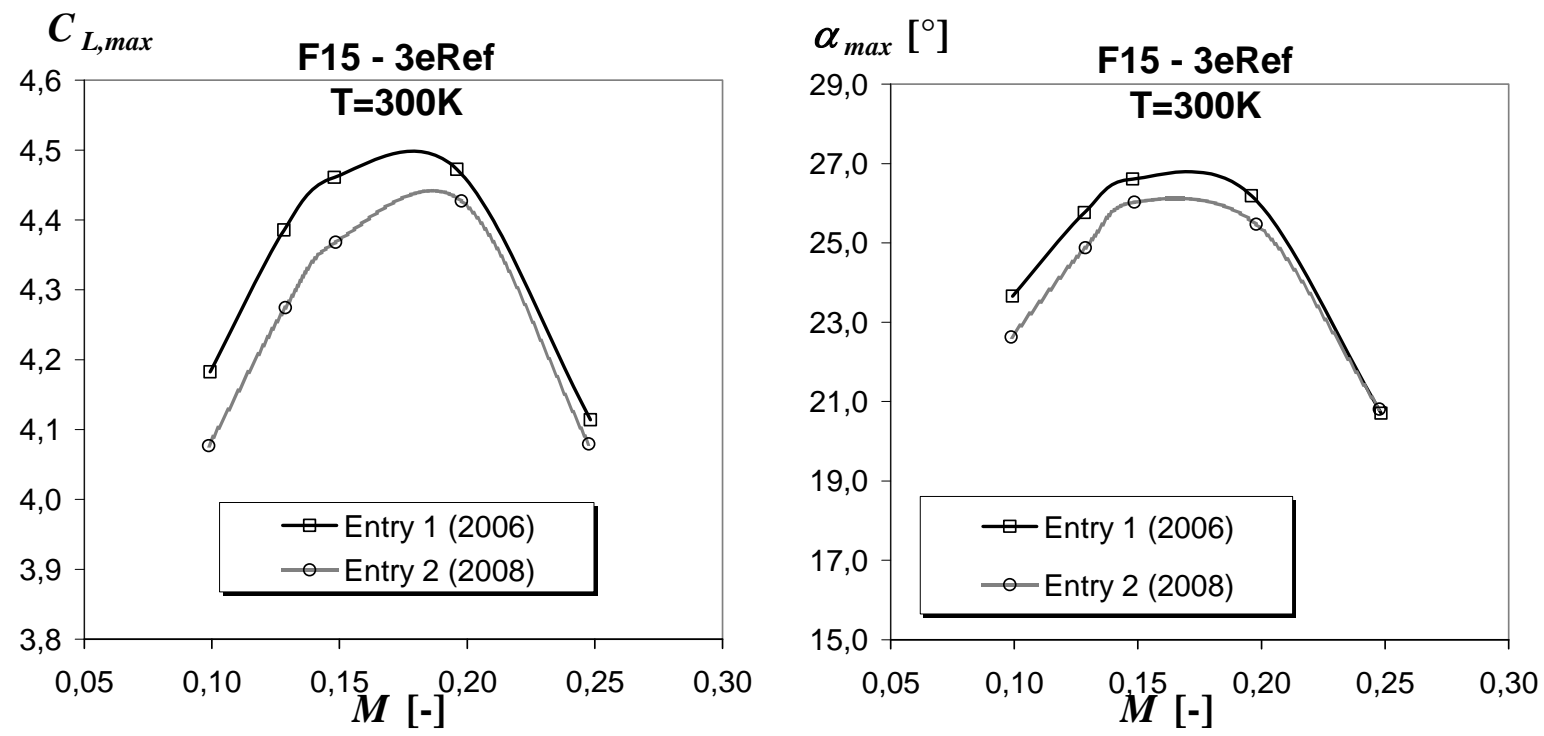

Figure 7. Long term repeatability of the stall characteristics of the DLR-F15 3eRef configuration at ambient conditions. 


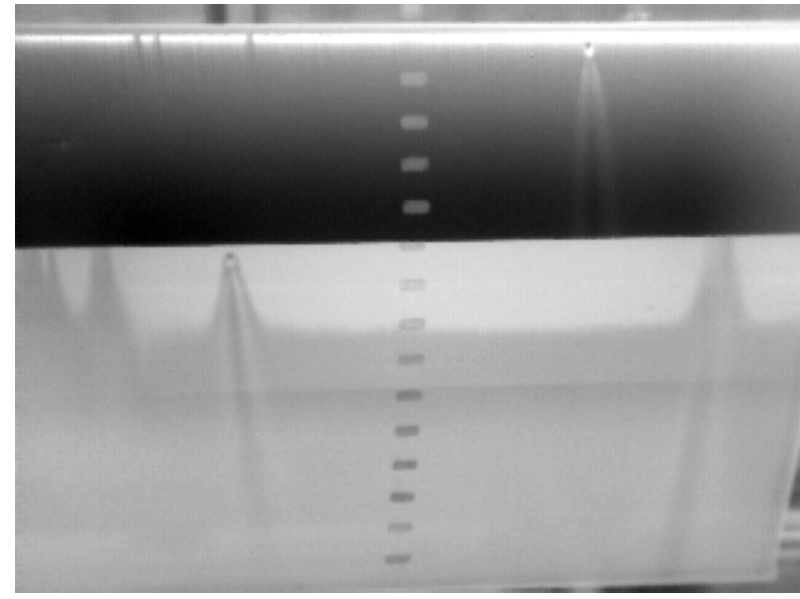

Figure 8. IR pictures of the suction side of the F15VLCS configuration visualizing laminar-turbulent transition.

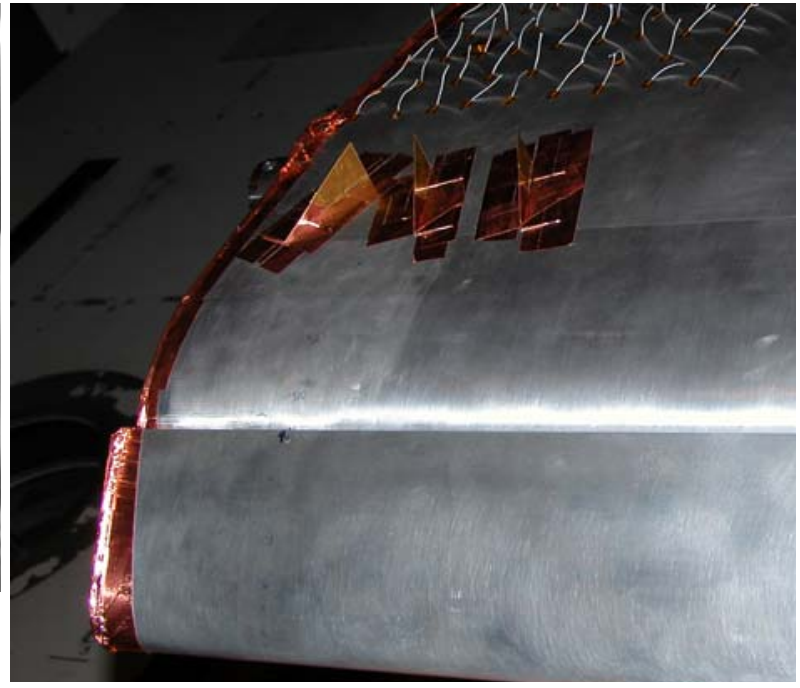

Figure 9. Vortex generator strake configuration used to prevent early side-wall stall.

\section{Results}

\section{A. Long term repeatability}

Within the first and the last campaign the F15-3eRef configuration was measured at ambient conditions. Figure 7 shows the stall characteristics in terms of maximum lift coefficient and corresponding angle of attack over the Mach number for ambient conditions $(T=300 \mathrm{~K})$. The shift is dedicated to some differences in the available pressure ports for the integration of the aerodynamic coefficients, reflected in a different level of maximum lift coefficients. The limited accuracy of absolute values has to be seen as a drawback of the method of pressure integration to determine aerodynamic coefficients, but is still believed to be accurate to determine increments obtained within one campaign. It seems not appropriate to directly compare data obtained within different campaigns. Beside this, the long term repeatability for the stall behavior itself seems good concerning the slope of the curves. The evolution of both the maximum lift coefficient and the corresponding angle of attack over the Mach number for constant ambient temperature agrees well between the two campaigns.

\section{B. Basic flow and improvements by side-wall stall prevention}

A crucial issue of measuring a 2D airfoil with a wall-to wall model is to check if the resulting flow is approximately 2D for a large span of the model, especially with respect to the center section, where pressure measurements are taken for the integration of the aerodynamic coefficients. Due to the difficult optical assessment in the DNW-KKK, previous tests in the DNW-NWB wind tunnel have been performed using infrared images to assess transition location. Figure 8 shows a view on the left hand side of the model (center section is right of the displayed area) of the F15-VLCS configuration at a high incidence of $\alpha=25^{\circ}$. The transition line on the main wing is clearly visible as well as the signature of vortex structures resulting from the brackets of the slat and discrete reference marker disturbances. The straight line indicates a well balanced 2D flow field over the major part of the model.

A major issue of two-dimensional wall-to-wall models, especially at high aerodynamic loads, is the appearance of a significant separation at the junction of model and wind tunnel wall originating from the boundary layer momentum losses along the tunnel side walls. This separation deteriorates the overall flow by channeling the remaining flow and resulting in unexpected effects on the resulting values, especially around the appearance of stall onset in the center section. In order to assess this, first the pressure distributions of the center section and the outboard sections have been compared. Configurations that stall very differently in these three sections cannot be assessed properly regarding their maximum lift potential, which is most likely over predicted in these cases.

Based on this experience, steps were taken to prevent an early side wall stall. In literature side wall suction or blowing has been used to improve the situation ${ }^{7}$. Nevertheless, it is known that this method is very hard to control in the sense that the achieved maximum lift of a $2 \mathrm{D}$ model strongly depends on the suction or blowing rates. Previous tests with the F15 model in another wind tunnel revealed that a specific arrangement of simple vortex 
F15 - 3eRef

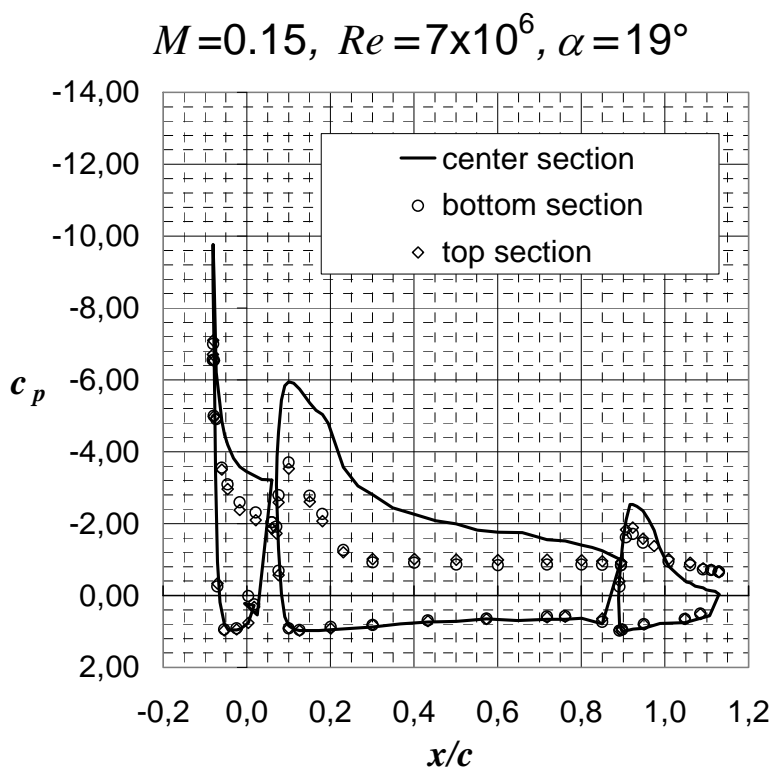

F15 - 3eRef (VG)

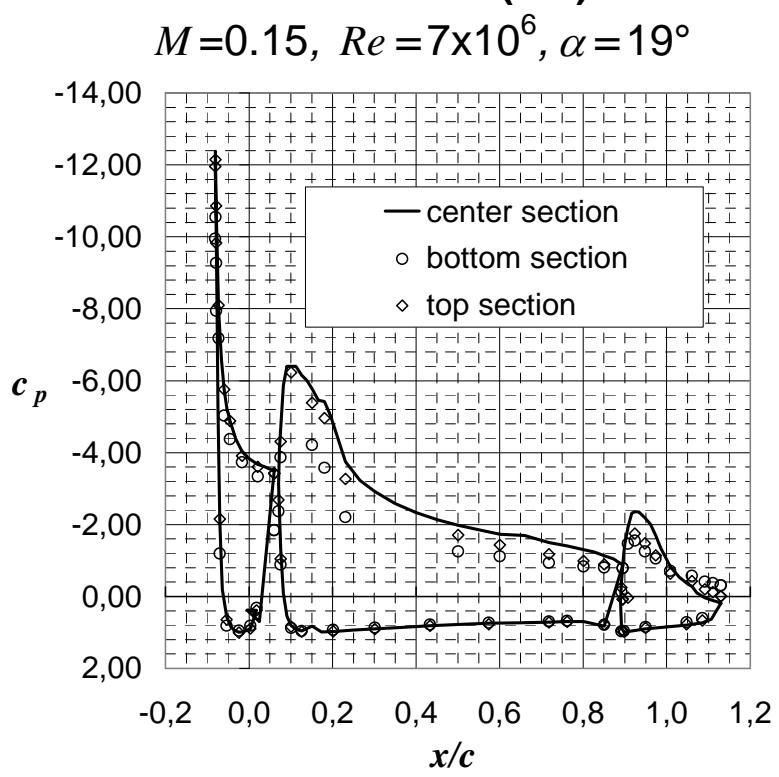

Figure 10.Comparison of center and side pressure distributions without (left) and with (right) vortex generator strakes to prevent early side-wall stall.

generators, shown in Figure 9 close to the side wall does a reasonable job in preventing side wall separation just far enough to get reasonable predictions of maximum lift.

Figure 10 shows pressure distributions for the configuration F15-3eRef at a high incidence of $\alpha=19^{\circ}$. Beside some residing differences the agreement over a span of $2.2 \mathrm{~m}$ is acceptable to assume $2 \mathrm{D}$ flow over the major part of the model. Nevertheless, of course the slat and flap brackets themselves and the special glove at the bottom wall needed to cover shrinkage of the model at low temperatures form a three-dimensional disturbance.

Figure 11 shows a comparison of flow fields perpendicular to the flow direction behind the model in configuration F15-3eRef at a high angle of attack of $\alpha=19^{\circ}$ with and without the vortex generators preventing the early side-wall stall. The large vortex structure close to the end of the model resulting from the side wall separation is clearly suppressed by the vortex generators. Due to the position of the vortex generators in the area of major flow acceleration the generated vortices scale in strength with the loading of the model and therefore the effect on preventing flow separation increases at the same time as the side-wall separation gets more likely. Another hint is the comparison of pressure distributions of the center and the outer sections.

However, the application of the vortex generators wasn't done in the vey first campaigns, since it was one of the outcomes of the first tests that an improvement can and should be made. For this reason they have not been applied to all configurations reported here. Measurements made with vortex generators are marked further on (VG), but for the same reason comparisons are not directly made within the same diagram for configurations with and without vortex generators. Nevertheless, earlier experience with the F15-model ${ }^{8}$ revealed that the use of the vortex generators does not influence the flow characteristics responsible for the stall behavior. They mainly avoid an unexpected non-linearity in the slope of the lift coefficient over the angle of attack, which had been visible as a decrease of the lift curve slope when side-wall separation developed. The suppression of the side-wall stall straightens the lift curve and stall occurs at lower angles of attack, but showing very similar pressure distributions. This is well according to theory, by which the stall onset on the outer wing mainly produces a span-wise lift distribution inducing a reduced angle of attack at the center section, and reducing the derivative of lift coefficient on angle of attack.

\section{Mach number dependencies of 3-element configurations}

Figure 12 shows the dependency of the maximum lift coefficients of all three 3-element configurations on the Mach number at two distinct Reynolds numbers, $R e=4 \times 10^{6}$ and $R e=7 \times 10^{6}$. For comparison, the dotted lines show the same dependency but at constant temperature and correspondingly increasing Reynolds number. All three 
configurations - although with different settings of both high-lift elements and even for the F15-VLCS a different size and shape of the slat - show a similar behavior. At low Mach numbers up to about $M=0.17$ the curves differ depending on the Reynolds number. The maximum lift coefficients obtained at the lower Reynolds number are higher than those at the higher Reynolds number. At Mach numbers between $M=0.17$ and $M=0.2$ the curves come close together and the difference due the Reynolds number almost disappears. Beside this, all configurations except for the F15-3eRef at $R e=4 \times 10^{6}$ and the F15-VLCS at $R e=7 \times 10^{6}-$ show a maximum at $M=0.15$. The first exception shows a continuous decay of maximum lift coefficient with increasing Mach number, the second also shows a maximum but at a higher Mach number $M=0.2$. At Mach numbers beyond $M=0.2$ the maximum lift coefficient significantly decreases for the two configurations measured at mach numbers up to $M=0.25$.

At this point it should be emphasized that the Mach number effect is not completely determined by measurements at constant temperature. The measurements where both the Mach and Reynolds number are varied at the same time show significant different behavior than those where the Reynolds number is approximately constant. Especially the measurements at ambient temperature show a completely different shape of the curves. It is very clear that this is only feasible in either pressurized or cryogenic wind tunnels, but otherwise it always will be hard to distinguish Mach and Reynolds number effects.
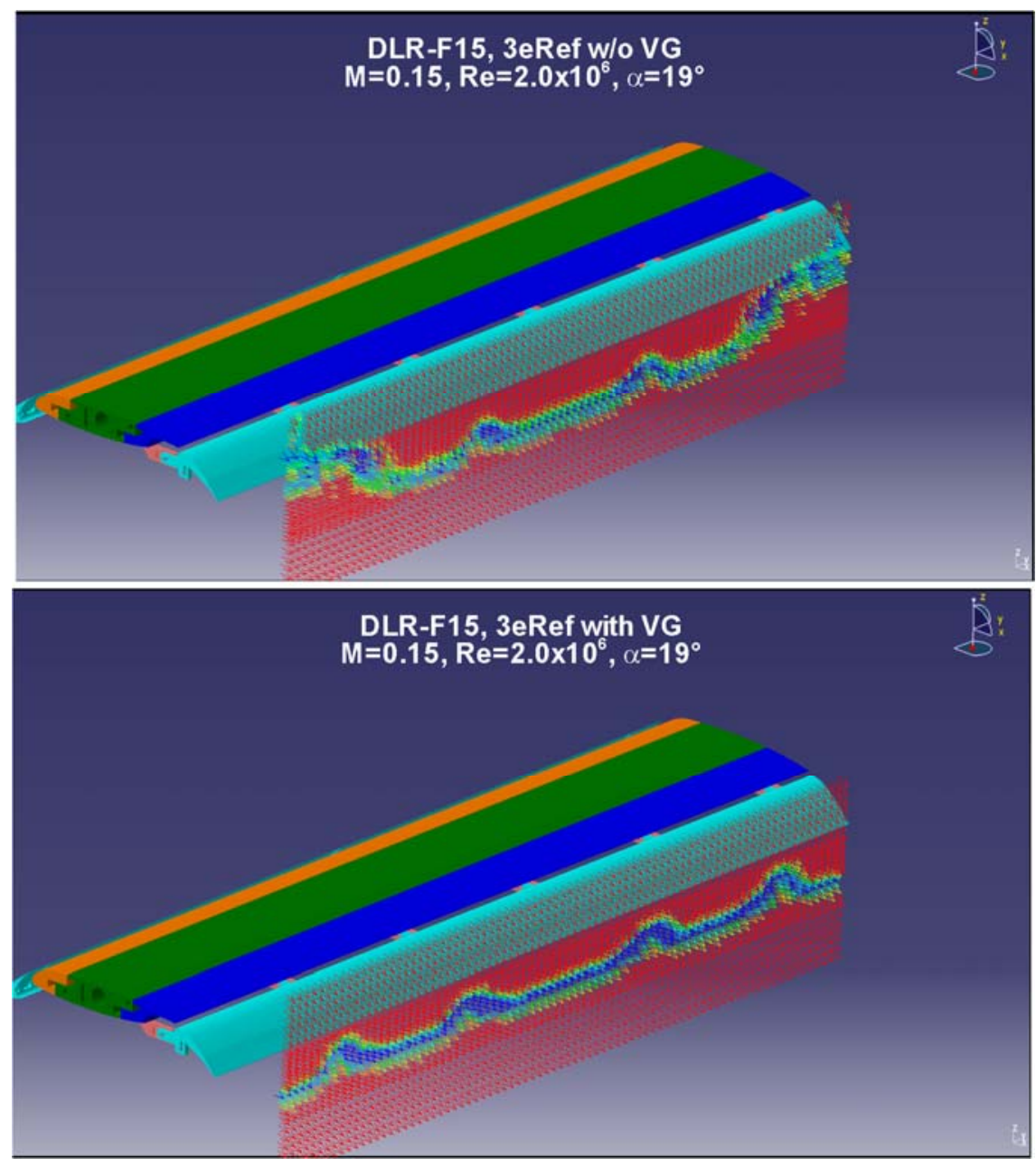

Figure 11.Comparison of flow field data by 5-hole probe rake measurements for the 3-element wing F15-3eRef at $\alpha=19^{\circ}$ with and without vortex generators for prevention of early side wall stall.

8

American Institute of Aeronautics and Astronautics 

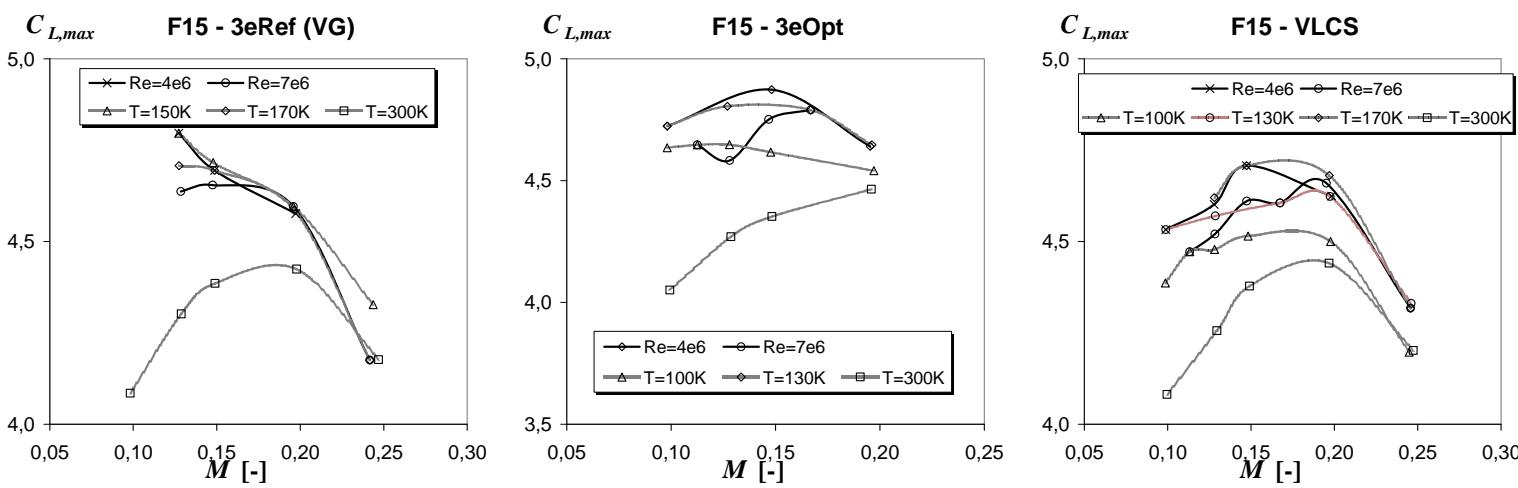

Figure 12.Dependency of maximum lift coefficient on the Mach number of all 3-element configurations at constant reynolds number compared to dependency at constant temperature.
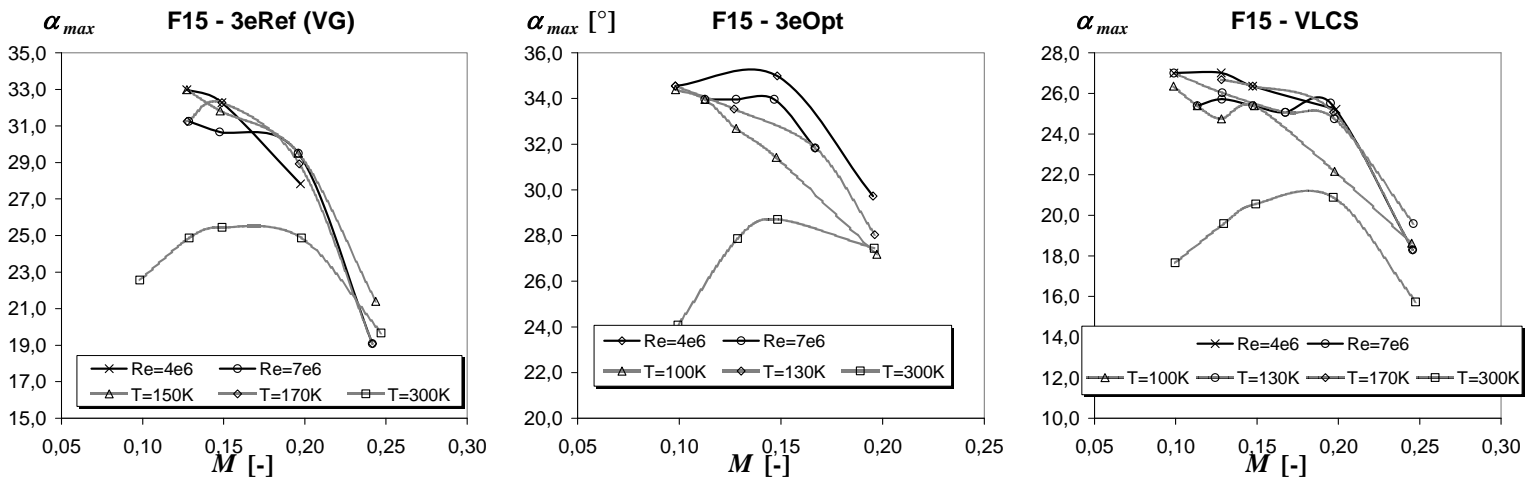

Figure 13.Dependency of the angle of attack for maximum lift coefficient on the Mach number of all 3element configurations at constant Reynolds number compared to dependency at constant temperature.

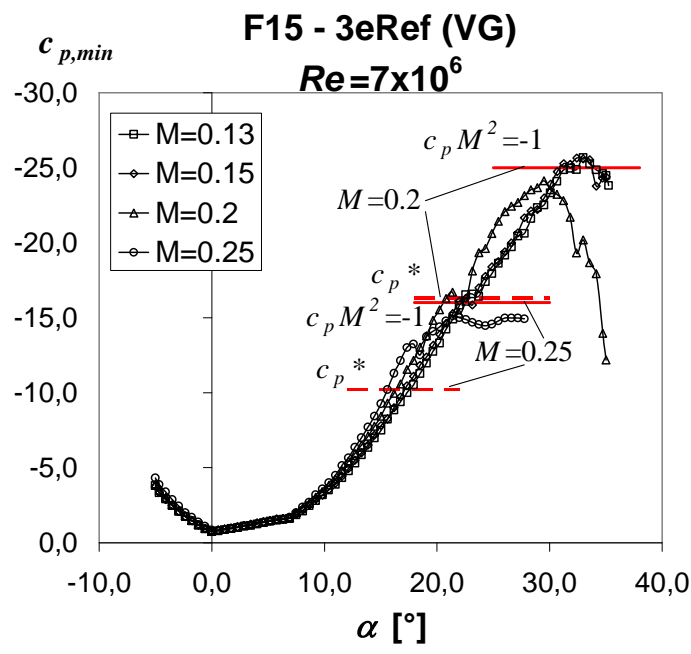

Figure 14.Evolution of the minimum pressure coefficient at the slat over the angle of attack for four different Mach numbers for the F15-3eRef configuration at $R e=7 \times 10^{6}$.
Regarding the angle of attack where maximum lift occurs (Figure 13) similar dependencies are observed as for the maximum lift coefficient itself. The curves seem kind of wavy, which is a result of the limited step size of between $\Delta \alpha=0.5^{\circ}$ and $\Delta \alpha=0.25^{\circ}$. The stall angle is almost of similar magnitude for Mach numbers below $M=0.15$ and is followed by a massive drop of about $10^{\circ}$ when increasing the Mach number from $M=0.2$ to $M=0.25$. Only the F15-VLCS at $R e=7 \times 10^{6}$ shows a significant decrease of stall angle not before $M=0.2$, corresponding to the maximum of maximum lift coefficient. This indicates that at higher Mach numbers stall the achievable maximum lift is directly linked to the related angle of attack, while at lower mach numbers the variation of the maximum lift coefficient is not related to a change of the corresponding incidence. A conclusion is that above $M=0.15$ the mechanism limiting the achievable maximum lift coefficient changes.

A deeper insight is obtained by looking on the development of the minimum pressure coefficient at the slat element. Figure 14 shows the evolution of this over the angle of attack for the F15-3eRef

American Institute of Aeronautics and Astronautics 
configuration at a Reynolds number of $R e=7 \times 10^{6}$. In the graph two known limits are introduced, which are in the scale of the graph only relevant for the two higher Mach numbers. The first is the critical pressure coefficient, where the flow locally gets supersonic. The second is the so called 0.7 vacuum limit according to measurements of Mayer ${ }^{9}$. Mayer showed in his experiments that with a certain accuracy there is no attached flow when $c_{p} M^{2}<-1$. Regarding the minimum pressure coefficient, the curves for the lower mach numbers $M=0.13$ and $M=0.15$ show no difference, while with increasing Mach number a compressibility effect gets visible for angles of attacks greater than $\alpha=10^{\circ}$. At $M=0.2$ the slope shows an intermediate break when reaching the critical pressure coefficient. At both higher Mach numbers the compressibility effect starts to stagnate and the minimum pressure coefficient is achieved only slightly below Mayer's 0.7 vacuum criterion. While for $\mathrm{M}=0.2$ reaching this upper

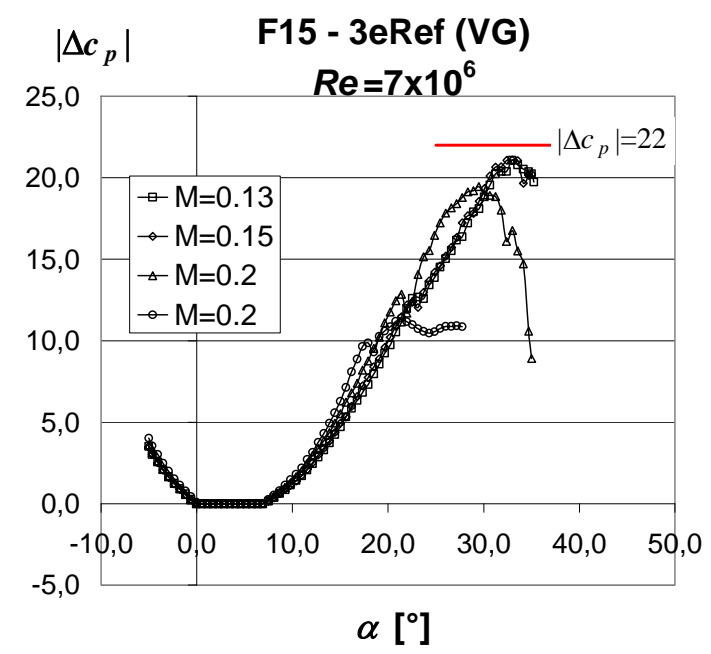

Figure 15.Evolution of the pressure rise at the slat over the angle of attack for four different Mach numbers for the F15-3eRef configuration at $R e=7 \times 10^{6}$.

limit corresponds to the onset of stall, indicated by

the decrease of minimum pressure level, for $\mathrm{M}=0.25$ the minimum pressure coefficient stays close to this boundary for the remaining range of measured incidences.

Another lift limit, which is some kind of agreed in the community although hard to assess the origin, is an assumed maximum allowable pressure rise over the slat and wing elements. Woodward and Lean ${ }^{10}$ state upper limits for the pressure rise $\left|\Delta c_{p}\right|=\left|c_{p, \min }-C_{p, T E}\right|$ over the slat of $\left|\Delta c_{p}\right|<22$, and of $\left|\Delta c_{p}\right|<11$ over the wing respectively. Figure 15 shows the evolution of the pressure rise over the slat for the F15-3eRef configuration again for the four measured Mach numbers at $R e=7 \times 10^{6}$. The pressure rise at low angles of attack between $\alpha=0^{\circ}$ and $\alpha=8^{\circ}$ is exactly zero, since for these configurations the minimum pressure is located at the trailing edge. The measurements verify the lift limit of $\left|\Delta c_{p}\right|<22$ with an acceptable accuracy for the two lower Mach numbers. Summarizing for this configuration, stall is triggered by the slat by approaching one or the other bounding limit. At lower Mach numbers the pressure rise limit is reached, while at higher Mach numbers the 0.7 vacuum limit is hit. Reaching the critical pressure coefficient reduces the additional rise of the pressure coefficient level due to compressibility, but does not directly induce stall onset.

While the F15-3eOpt shows a similar behavior for the slat, for the F15-VLCS configuration slight differences are visible. Figure 16 shows both the evolution of the minimum pressure at the slat and the pressure rise for the F15-

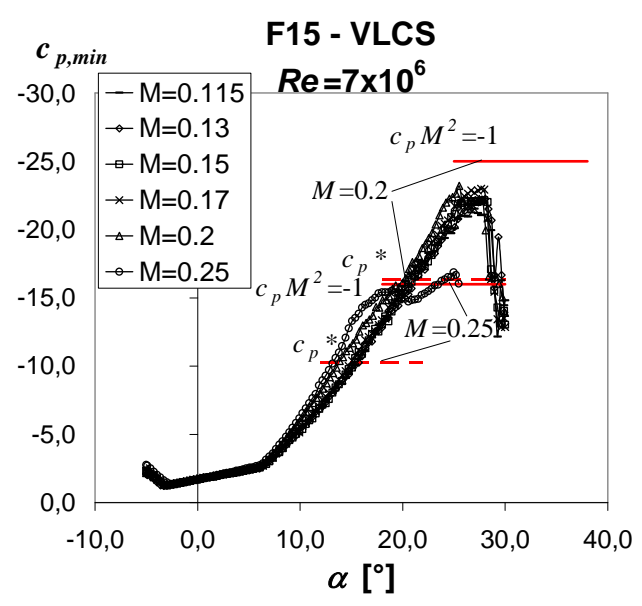

(a)

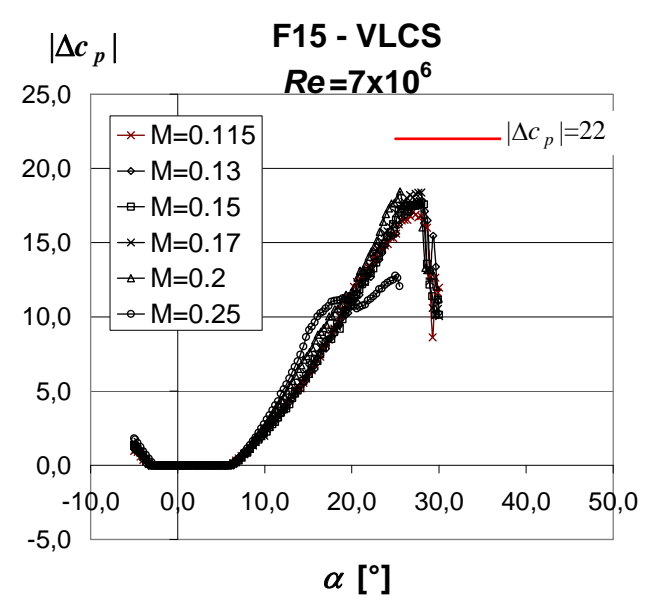

(b)

Figure 16.Evolution of the (a) minimum pressure coefficient, and (b) pressure rise at the slat over the angle of attack for six different Mach numbers for the F15-VLCS configuration at $R e=7 \times 10^{6}$. 


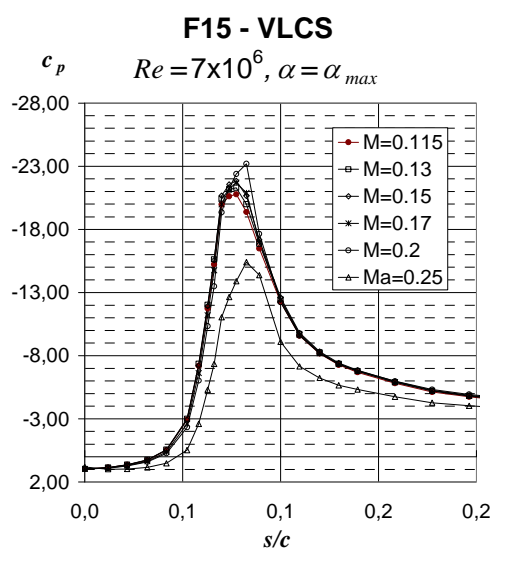

(a)

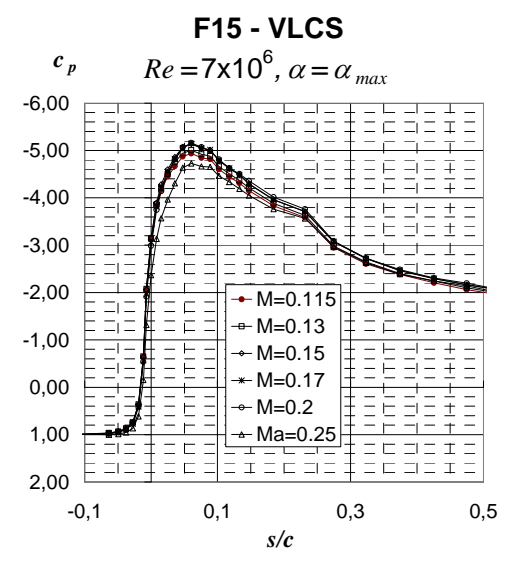

(b)

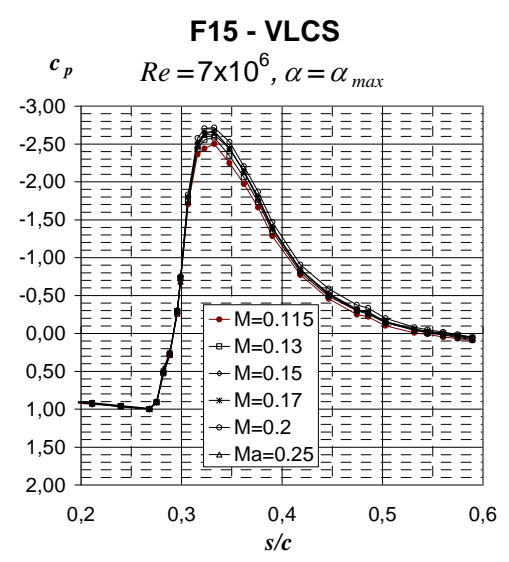

(c)

Figure 17.Pressure coefficient distribution along the local arc length along (a) the slat, (b) the wing leading edge, and (c) the flap at the angle of attack of maximum lift coefficient in dependence of the Mach numbers for the F15-VLCS configuration at $R e=7 \times 10^{6}$.

VLCS. Although for $\mathrm{M}=0.2$ the compressibility effect is clearly visible the minimum pressure level does not reach up to the minimum pressure bound but stalls at similar levels as for the lower Mach numbers. The plot of the pressure rise reveals that even the pressure rise criterion is not achieved and the maximum pressure rise seems to be limited to a value around $\left|\Delta c_{p}\right|<18$. It is a straight forward conclusion that the latter limit cannot be seen as an absolute value but is somehow related to relative chord length of the slat element. Since for this configuration the pressure rise limit is hit earlier than the 0.7 vacuum limit, the above mentioned shift of the maximum achievable maximum lift coefficient to the higher Mach number can be explained.

Summarizing the effects on the slat element, both limits play an important role. At lower Mach numbers the pressure rise limit is more crucial, but the overall maximum lift has still a slightly increasing tendency up to the Mach number, where the minimum pressure limit becomes significant.

Nevertheless, the explanation for the increasing maximum lift coefficient at low Mach numbers is still missing. Since the characteristics at the slat investigated so far don't reveal significant differences, a look has to be taken on the other elements. Figure 17 displays details of the pressure coefficient distribution over the local arc length along the airfoil elements at the angle of attack corresponding to the maximum lift coefficient for the measured Mach numbers of the F15-VLCS configuration at $R e=7 \times 10^{6}$. For the slat and the flap the arc length is counted starting from the lower trailing edge, along the lower side, around the nose, and along the upper side to the trailing edge. For the main wing the arc length zero coordinated is shifted to the leading edge, thus negative values representing the lower side. Aside the pressure distributions for the highest Mach number $M=0.25$, all elements show a slightly increasing suction pressure level with increasing Mach number. At $M=0.2$ the development of the shock at the slat leading edge is visible. Although of no big severity, the increase of suction pressure level can only be explained by the onset of compressibility effects, even for the very low Mach number of $M=0.115$. Reminding that the local
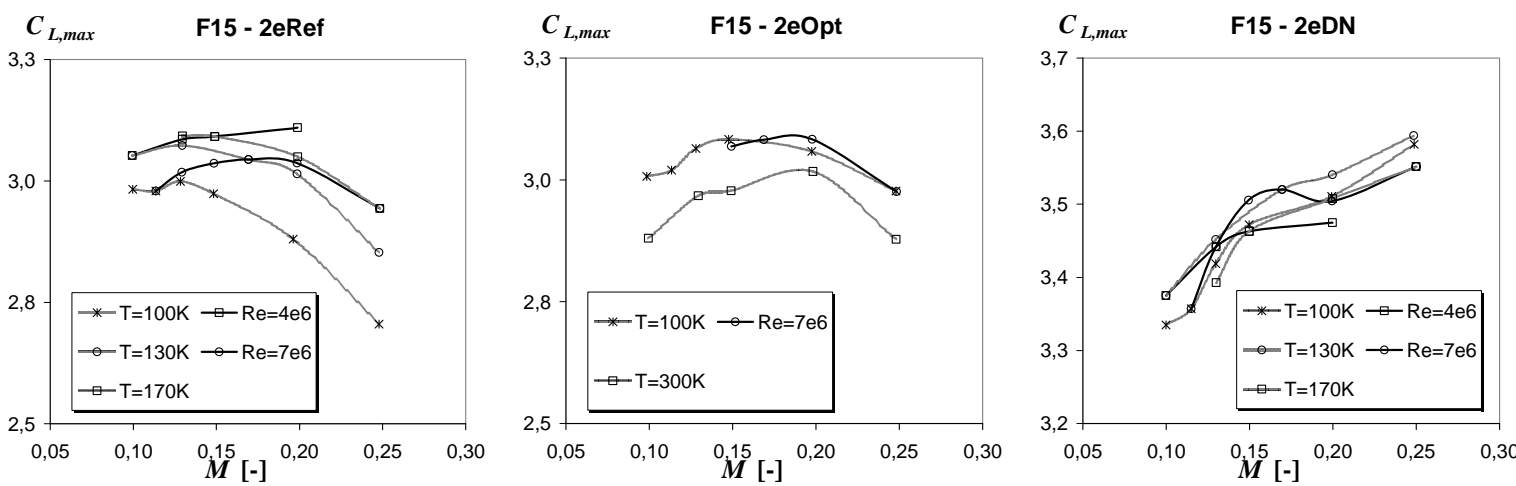

Figure 18.Dependency of maximum lift coefficient on the Mach number of all 2-element configurations at constant Reynolds number compared to dependency at constant temperature. 


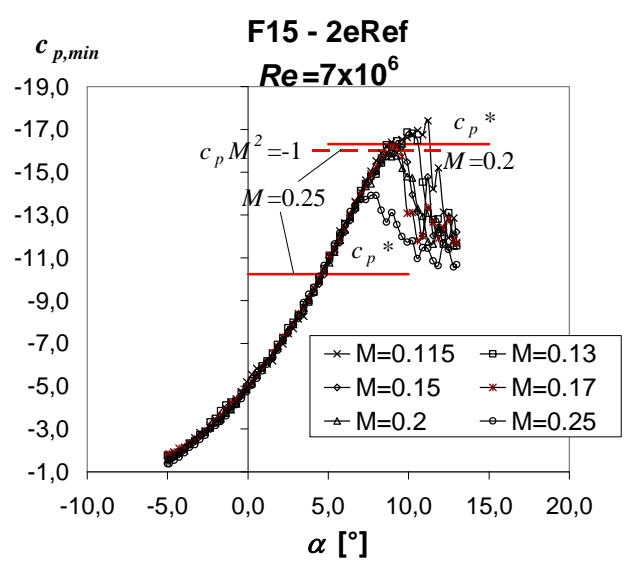

(a)

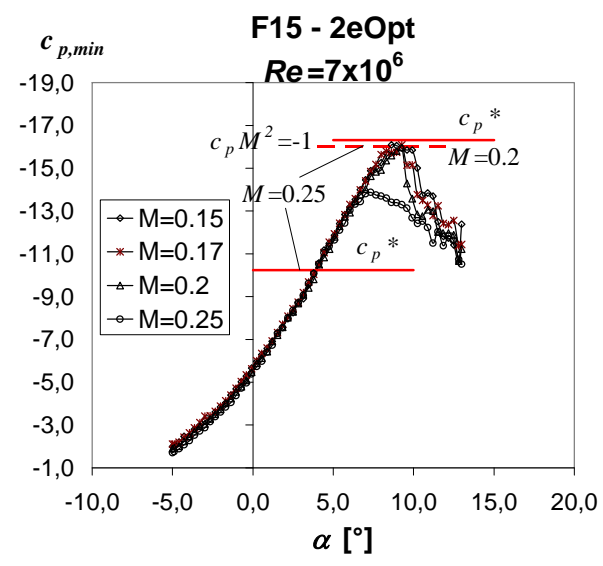

(b)

Figure 19. Evolution of the minimum pressure coefficient at the main wing over the angle of attack for four different Mach numbers for the (a) F15-2eRef and (b) F15-2eOpt configurations at $R e=7 \times 10^{6}$.

velocity ratio is linked to the square root of the pressure coefficient, approximately the same holds for the Mach number, thus the local Mach number at the slat being more than 4.5 times the Mach number and the local Mach number is exceeding the agreed limit for incompressible flow of $M=0.3$.

\section{Mach number dependencies of 2-element configuration}

Before discussing the effects of the Mach number on the stall behavior of the investigated 2-element configurations, it has to be mentioned that most of the data available has been produced without vortex generators. In detail, the measurements with vortex generators are too few to allow conclusions.

Figure 18 shows the behavior of the maximum lift coefficient of all three 2-element configurations for increasing Mach number at constant Reynolds number, again with comparison to measurements at constant temperature. Compared to the 3-element configurations the maximum lift coefficient has an increasing tendency up to a Mach number $M=0.2$ for all configurations. The maximum left breakdown towards $M=0.25$ is not as severe as for the 3-element configurations. Interestingly, the droop nose configuration F15-2eDN shows no maximum lift break down even at $M=0.25$, but a much steeper rise at low Mach numbers. Again, for the F15-2eRef it is most obvious that the Mach number trend cannot be correctly captured by measurements at constant temperature. At constant temperature the curves show a very early maximum at about $M=0.13$ and a decreasing slope for the following increase of the Mach number.

Figure 19 shows the evolution of the minimum pressure at the main wing element of the F15-2eRef and F15-

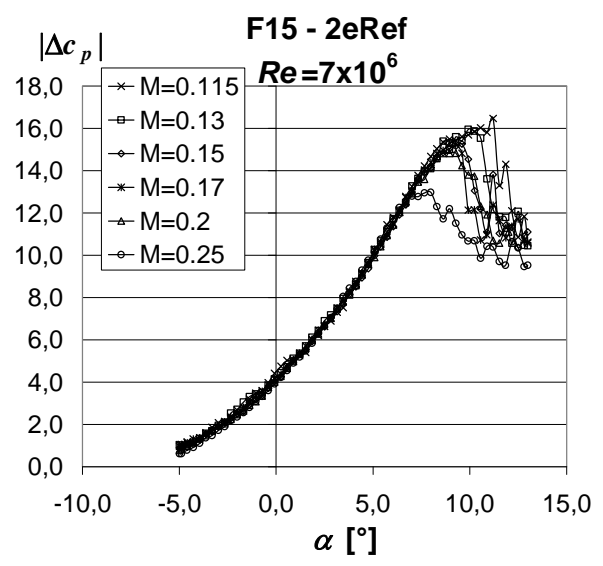

(a)

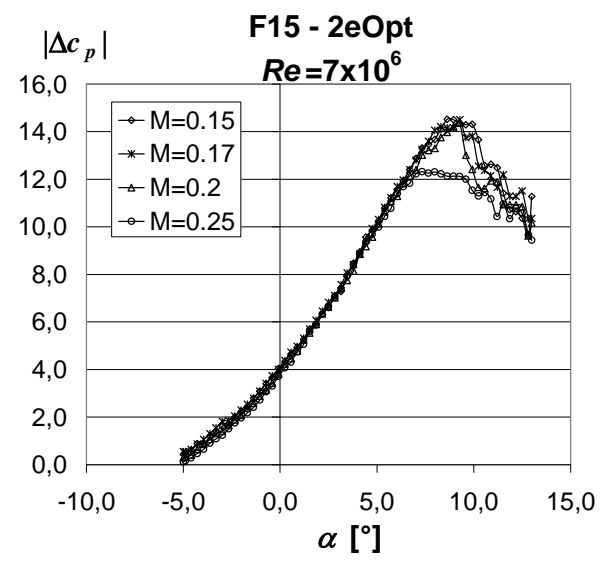

(b)

Figure 20. Evolution of the pressure rise at the main wing over the angle of attack for four different Mach numbers for the (a) F15-2eRef and (b) F15-2eOpt configurations at $R e=7 \times 10^{6}$.

American Institute of Aeronautics and Astronautics 


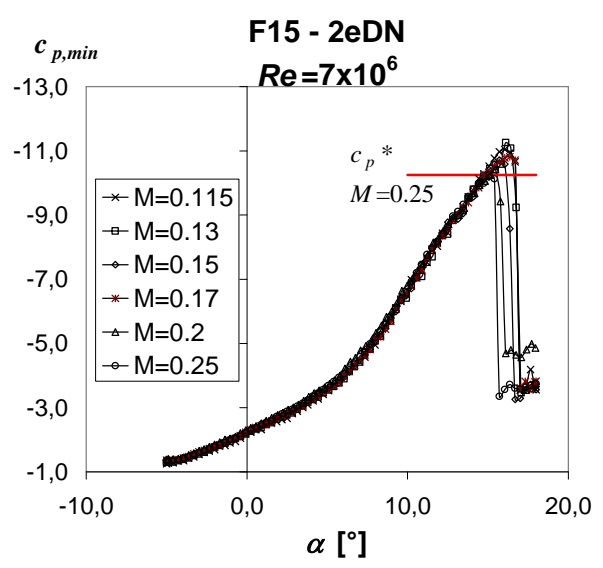

(a)

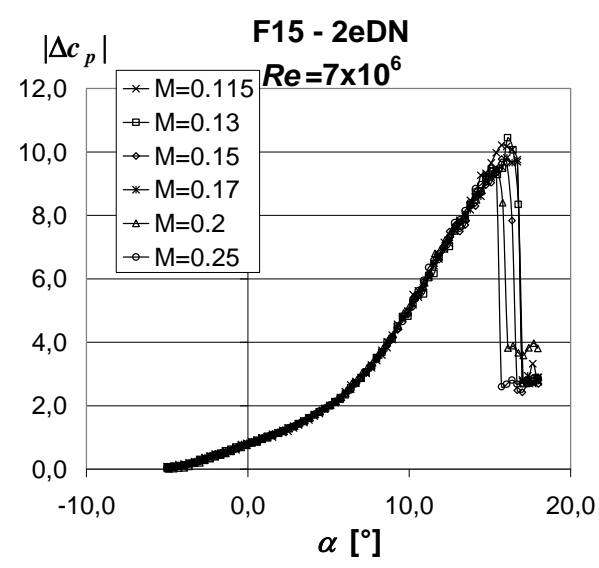

(b)

Figure 21.Evolution of the (a) minimum pressure coefficient, and (b) pressure rise at the main wing over the angle of attack for six different Mach numbers for the F15-2eDN configuration at $R e=7 \times 10^{6}$.

2eOpt configurations at $R e=7 \times 10^{6}$. For both configurations an undershoot of the critical pressure is only visible for the highest Mach number $M=0.25$. The minimum pressure limit is not touched at any Mach number shown here, but the minimum pressure achieved is limited around $c_{p}=-16$, independent of the flap setting.

Interestingly, the maximum pressure rise is dependent on the flap setting, as shown in Figure 20. The pressure rise obtained for the F15-2eRef is limited - although not as clearly - at about $\left|\Delta c_{p}\right|=16$, while the F15-2eOpt shows its maximum pressure rise already at about $\left|\Delta c_{p}\right|=14$. Deeper analysis unveils that this is solely related to the difference of the wing trailing edge pressure, which is less negative for the F15-2eRef configuration due to a flap separation, which is not developed for the F15-2eOpt. Nevertheless, up to now the F15-2eRef configuration is the only one up to now that shows a Mach number dependency on the minimum pressure and the pressure rise with a decreasing minimum pressure level or maximum pressure rise when increasing the Mach number from $M=0.115$ to $M=0.15$.

The last investigated configuration, F15-2eDN is expected to show a different stall behavior, since the smart droop nose shape is primarily designed to delay stall onset. The bending of the leading edge is designed to reduce the very high and sharp suction peak and therefore reduce the load on the boundary layer. Figure 21 shows the minimum pressure coefficient and the drag rise for this configuration. Just for the highest Mach number $M=0.25$ locally sonic speed is reached at the leading edge. This is surely the major reason, why this configuration shows no

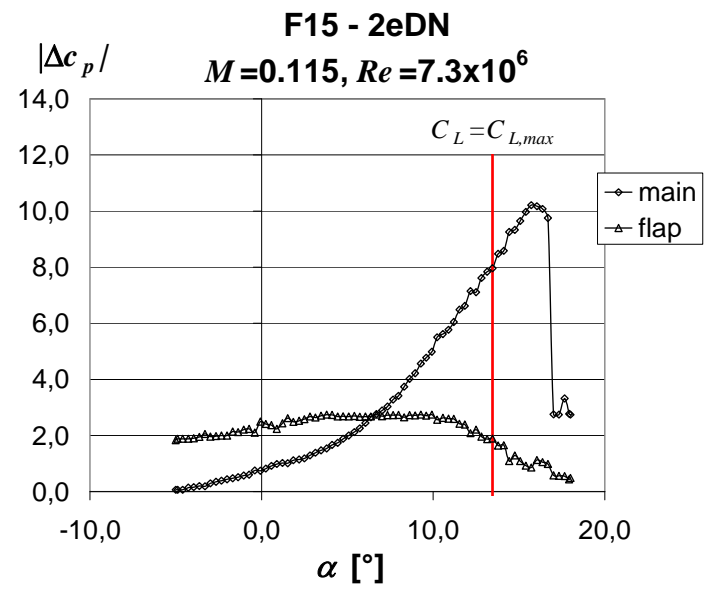

(a)

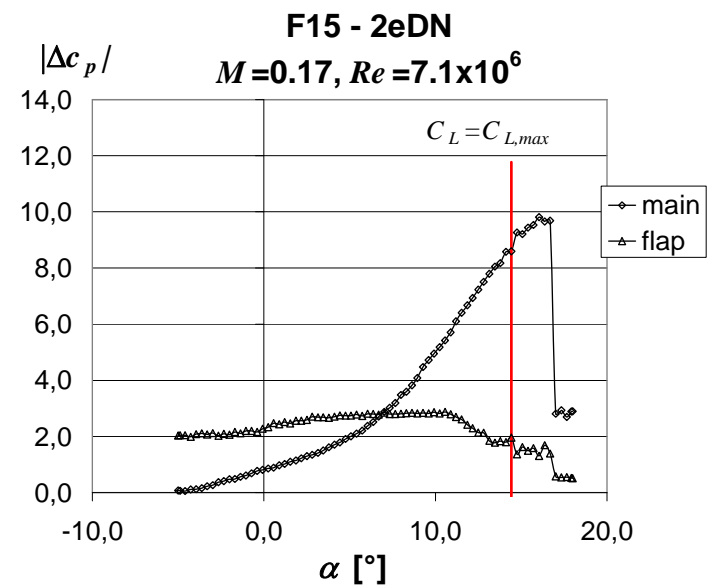

(b)

Figure 22.Evolution of the pressure rise at both elements of the F15-2eDN configuration at $\operatorname{Re}=7 \times 10^{6}$ over the angle of attack for (a) $M=0.115$, and (b) $M=0.17$. 
F15 - 2eRef

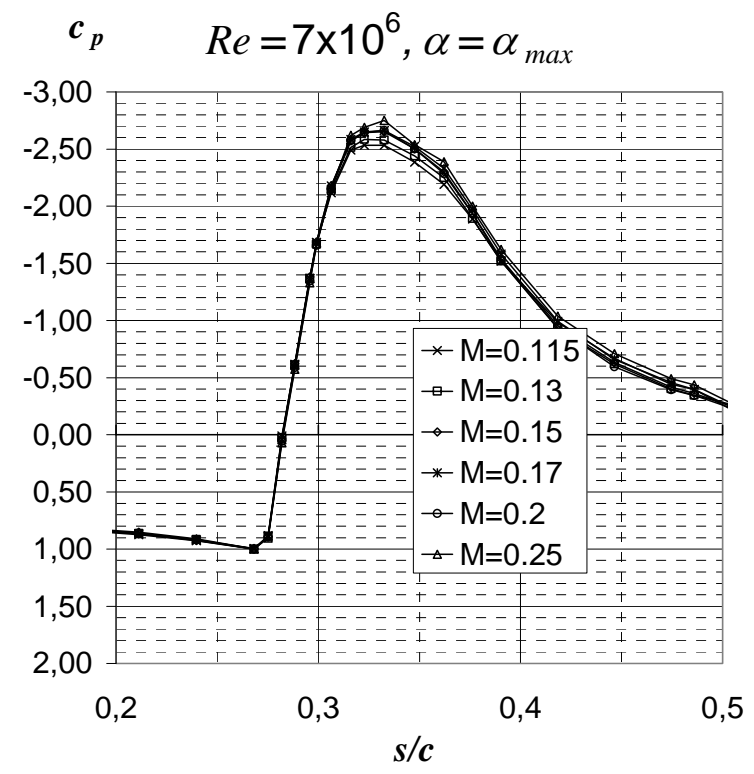

(a)

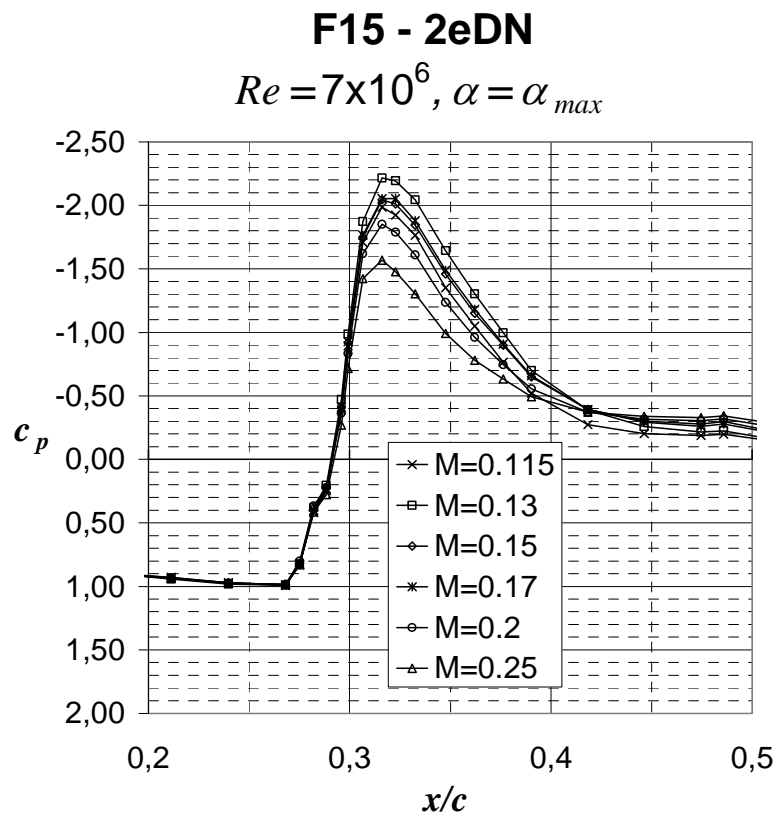

(b)

Figure 23.Variation of the pressure coefficient distribution with the Mach number at the flap leading edge at the angle of attack of maximum lift coefficient at $R e=7 \times 10^{6}$ for the (a) F15-2eRef, and (b) F15-2eDN configuration.

decrease in maximum lift value over the Mach number. The minimum pressure level as well as the maximum pressure rise decrease with increasing Mach number. On the first view, this is contradictory to the increasing maximum lift coefficient with increasing Mach number.

The explanation is only found in conjunction with an additional deeper look to the effects at the flap. For two Mach numbers the development of the pressure rise over both the main wing and the flap is shown in Figure 22, with the angle of attack marked where the maximum lift coefficient occurs. The graphs show that for this configuration the maximum lift coefficient is achieved well before the main wing stalls due to a separation on the flap itself. In contrast to the other configurations reported before - although not shown in detail - where the flap flow is attached at maximum lift, this configuration has a separated flap over the complete range of angles of attack. Therefore the maximum lift is achieved during an increasing load on the wing and a decreasing load on the flap. For higher Mach numbers the balanced maximum lift is closer to the maximum pressure rise than for lower Mach numbers.

The significant difference of the flap pressure distribution between the F15-2eDN and the F15-2eRef

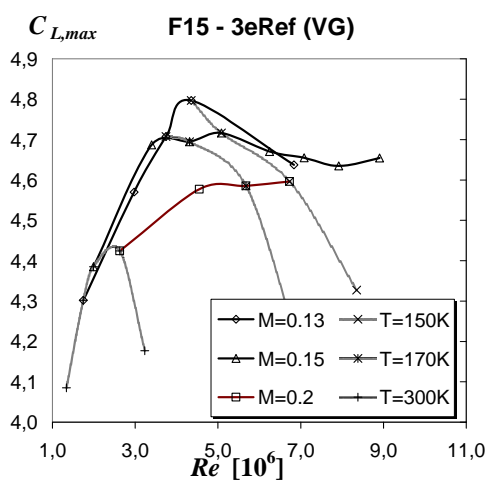

(a)

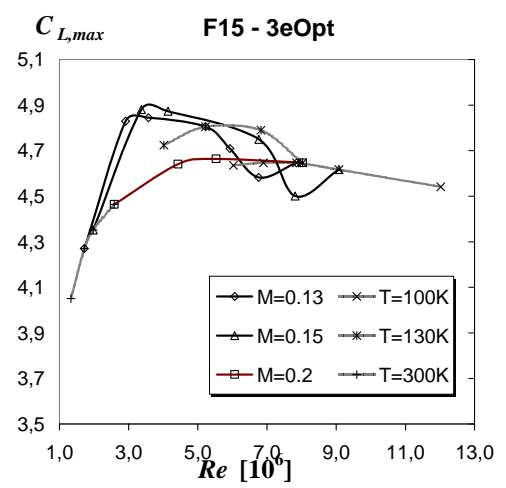

(b)

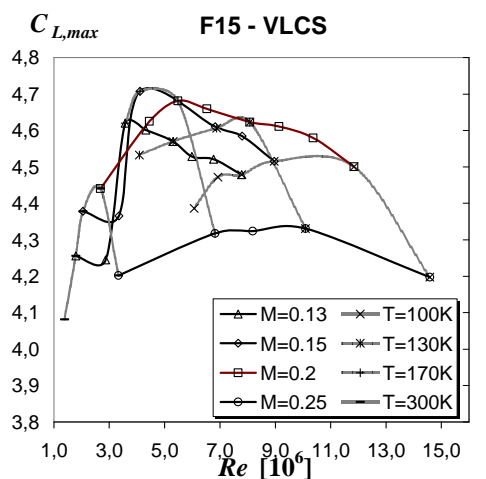

(c)

Figure 24.Dependency of maximum lift coefficient on the Reynolds number of all 3-element configurations at constant Mach number compared to dependency at constant temperature. 


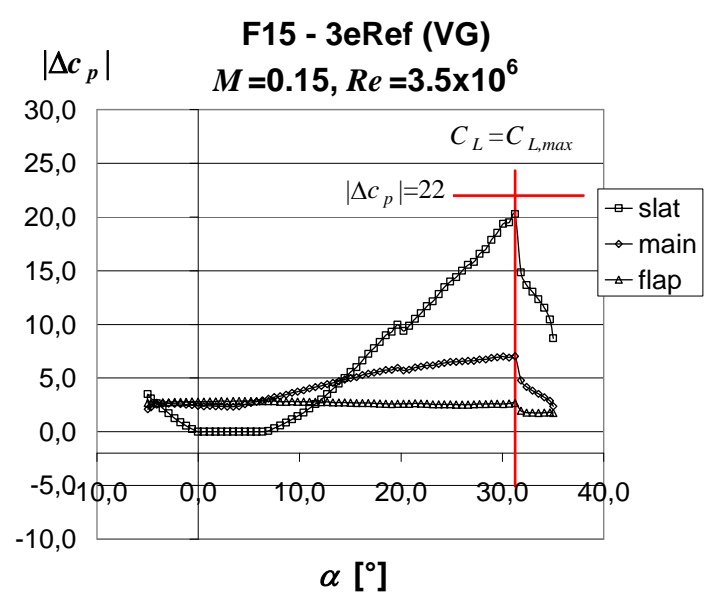

(a)

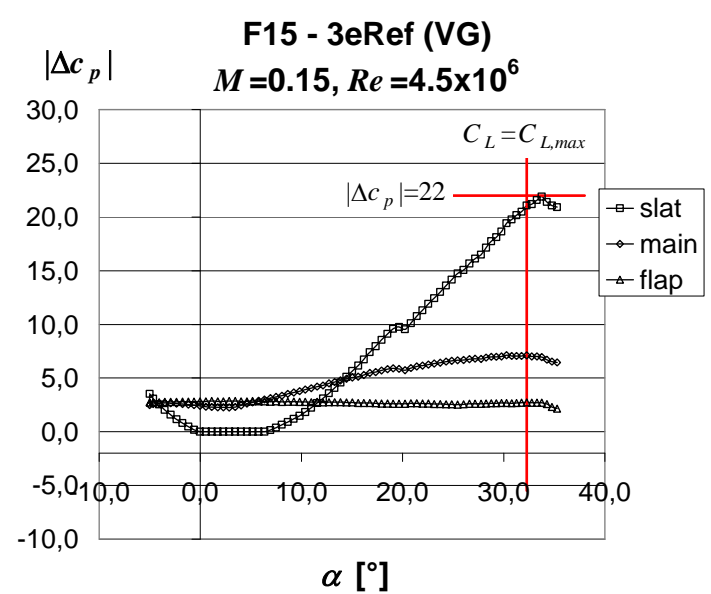

(b)

Figure 25.Evolution of the pressure rise at all three elements of the F15-3eRef configuration at $M=0.15$ over the angle of attack for (a) $R e=3.5 \times 10^{6}$, and (b) $R e=4.5 \times 10^{6}$.

configurations just at the angle of attack of maximum lift coefficient is shown in Figure 23. While the F15-2eRef shows an attached flap flow the F15-2eDN is separated. The separation on the flap is hereby sensitive to the Mach number, showing a decreasing suction pressure level with increasing Mach number. Nevertheless, the Mach number dependency of the flap separation is not yet fully understood and needs further analysis.

\section{E. Reynolds number dependencies of 3-element configurations}

Figure 24 shows the characteristics of the maximum lift coefficient over Reynolds number for the three different slat configurations F15-3eRef, F15-3eOpt and F15-VLCS at various Mach numbers. Additionally, again the relations at constant temperature, thus varying Mach and Reynolds number at the same time are shown. All three configurations show a steep increase of maximum lift coefficient up to a Reynolds number between $R e=3 \times 10^{6}$ and $R e=5 \times 10^{6}$ and a decreasing slope further on. The largest deviations - and therefore the highest sensitivity on the Reynolds number - are seen for the F15-VLCS configuration. At the highest Mach number of $M=0.2$ the Reynolds number sensitivity is less pronounced, which was already suggested during the discussion of the Mach number effects. The slope of the curves obtained at constant temperature show totally different sensitivity, and - again -a proper distinction of Reynolds and Mach number effects on the high-lift airfoil configuration cannot be done at constant temperature. The angle of attack for maximum lift coefficient - not shown here - shows a similar behavior as the maximum lift coefficient itself. The variation, especially the rise at low Reynolds numbers, is therefore not a result of minor effects of changing pressure levels.

For a deeper analysis, again a look at the evolution of characteristic values of the pressure distribution and to the pressure distributions just at maximum lift coefficient is necessary. At first, Figure $\mathbf{2 5}$ shows the pressure rise for all three elements of the F15-3eRef configuration at $M=0.15$ for the two Reynolds numbers $R e=3.5 \times 10^{6}$, and $R e=4.5 \times 10^{6}$. The stall behavior is significantly different. While for the lower Reynolds number the stall onset occurs on all three elements at the same angle of attack, at the only slightly higher Reynolds number the maximum lift is characterized by a stagnating pressure rise on the main wing element. While the first behavior looks like a leading edge separation type the second seems to be a smooth wing trailing edge separation onset. Pressure distributions for these two cases at and beyond the maximum lift incidence (Figure 26) reveal no evidence of either a leading edge separation or a laminar separation bubble burst for the lower Reynolds number. The pressure distributions show flow separation on half of the main wing chord within an incidence of only half a degree above maximum lift triggering also a complete flap separation. For the higher Reynolds number even four degrees above maximum lift only a small trailing edge separation on the main wing is visible and the both high-lift system elements show nearly no change. 
F15 - 3eRef (VG)

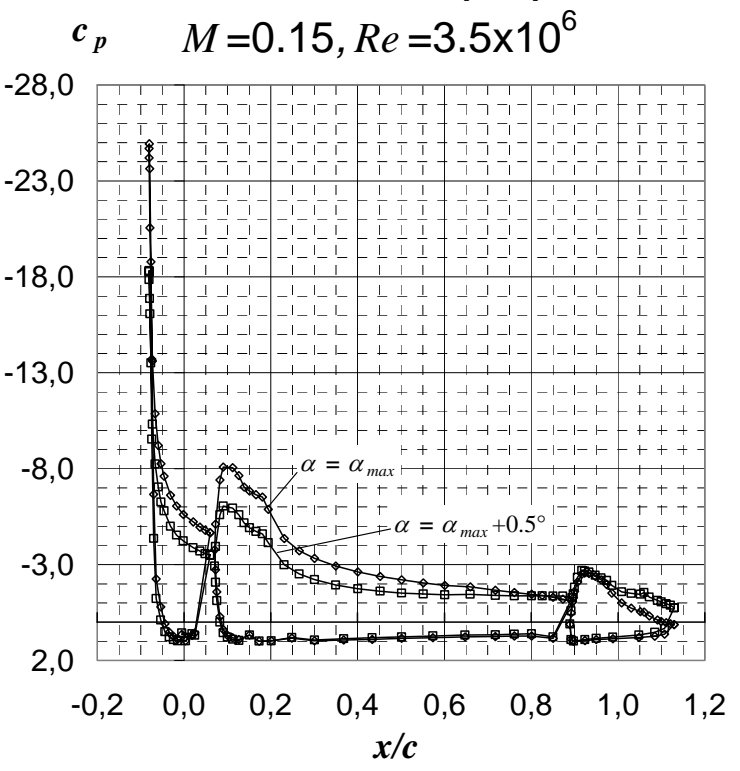

(a)
F15 - 3eRef (VG)

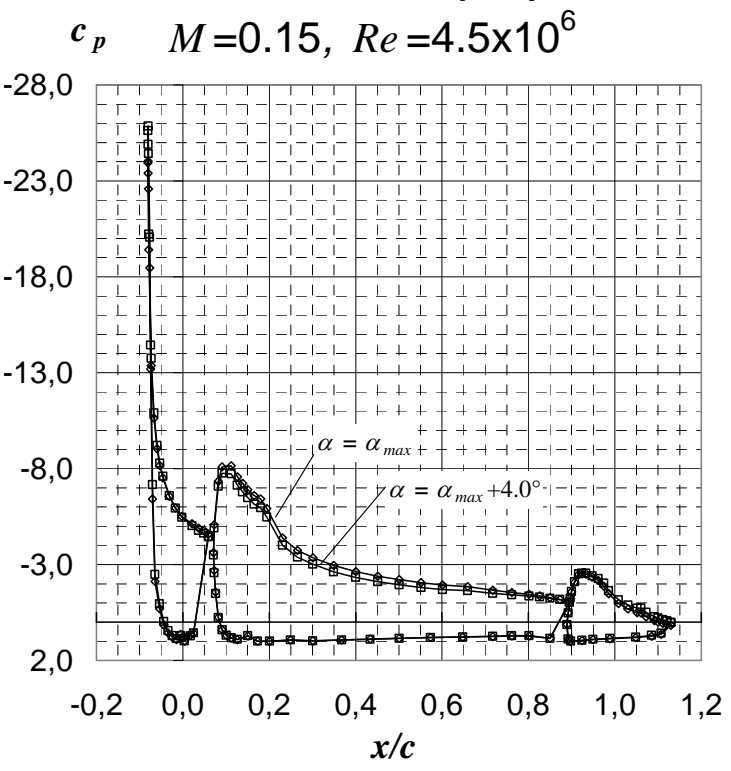

(b)

Figure 26.Variation of the pressure coefficient distribution of the F15-3eRef configuration at the angle of attack of maximum lift coefficient and beyond at $M=0.15$ and (a) $R e=3.5 \times 10^{6}$, and (b) $\operatorname{Re}=4.5 \times 10^{6}$.

From the data available there is no direct explanation for the sudden change of stall characteristics between $R e=3.5 \times 10^{6}$ and $R e=4.5 \times 10^{6}$. But it is clear that it is not the switch from a leading edge separation to a trailing edge separation like it is likely for single airfoils at this Reynolds number range. Since this can be excluded, the remaining mechanism is likely to reside in the mechanisms of wake development of the slat and its interaction with the wing boundary layer. It is explainable that at the lower Reynolds number thicker slat wakes develop, which should merge with the wing boundary layer more upstream than at higher Reynolds numbers. It is a straight conclusion to assume that an earlier merge must lead to an earlier trailing edge separation due to the velocity deficit at the out bound of the boundary layer of the wing, resulting in the earlier breakdown of maximum lift the lower the Reynolds number. Once the slat wake doesn't start to merge with the wing boundary layer upstream of the wing trailing edge, the lift breakdown is vanished. We performed some CFD analysis on this topic giving some confidence in this assumption, but an experimental proof of this theory has not yet been performed and would need additional more detailed field measurements to detect the wake-boundary layer merging. Unfortunately the slat setting of the F15-3eOpt configuration is not very different to the F15-3eRef settings, so it is not suitable for verification, and the F15-VLCS is too different in shape to draw direct conclusions of this kind. 
A second effect visible for all three configurations in the Reynolds dependency of the maximum lift coefficient is the decrease of maximum lift beyond the above discussed critical Reynolds number. This also needs some explanation since it is contradictory from what is known from single element airfoil theory. From theory with increasing Reynolds number the viscous displacement should reduce and should result in increasing maximum lift values asymptotically approaching a limit value for the Reynolds number towards infinity. For the 3-element airfoils the displacement of the flow through the gaps induced by the boundary layers gets more important, and not only for the stall behavior itself. Starting from the rear for the F15-3eRef and the F15-3eOpt configurations totally opposite effects are observed for the flap. Figure 27 shows the pressure distributions for both configurations at $M=0.15$ at the angle of attack of maximum lift coefficient at various Reynolds numbers. While the F15-3eRef configuration shows an increasing suction with increasing Reynolds number, the F15-3eOpt shows the opposite. Here the suction is decreasing with increasing Reynolds number. The difference in flap setting of both configurations is that the F153eOpt configuration has a smaller gap, a larger overlap, and a higher deflection angle than the F15-3eRef flap. But the main effect seen here is the role of the boundary layer displacement in the gap area itself. The F15-3eRef configuration shows the behavior how it can be derived from theory ${ }^{11}$ that an increased effective gap leads to a higher flap loading, similar to putting the flap slightly more away from the wing. For the F15-3eOpt with its small gap the gap blockage has a bigger influence. The gap blockage is reduced with increasing Reynolds number and the circulation effects develop, leading to a larger influence of the main wing on the flap and a thus reduced flap loading with increasing Reynolds number.

The same effects take place at slat. They are best observed looking at the pressure distributions on the slat and the main wing of the F15-VLCS configuration, where the maximum lift coefficient decrease is most significant. Here for both configurations the gaps are similar so the effect is the same. Figure $\mathbf{2 8}$ shows the pressure distribution along the local arc length of the slat and a zoom on the suction peak on the main wing of the F15-VLCS configuration at $M=0.15$. For clarity only three Reynolds numbers spanning the range of maximum lift coefficient decrease are shown. A continuous decrease of the suction level with increasing Reynolds number is visible. Already the graphs on optimum slat positioning shown by Woodward and Lean ${ }^{10}$ show a distinct optimum position of the slat for maximum lift coefficient, with a high sensitivity on the gap. The pressure distribution and the resulting maximum lift potential is always a balance between the upstream effect of the wing onto the slat and the downstream effect vice versa. Reducing the slat gap first brings the slat more into the influence of the wing, thus increasing the slat circulation. This increased circulation reduces the wing suction and therefore reduces again the circulation of the slat. Coming from an optimum position this means, that even for closing or opening the gap, the

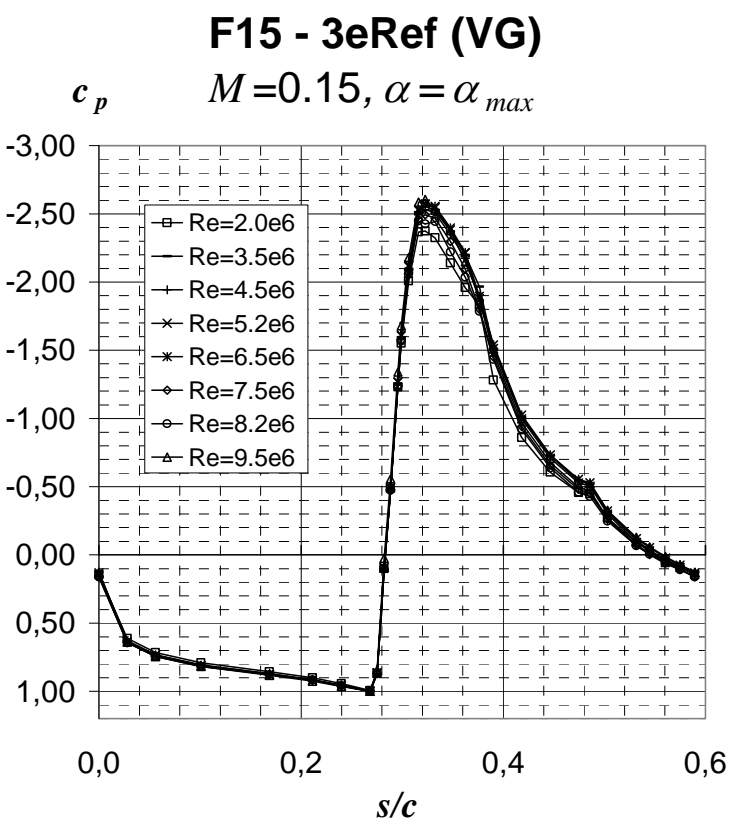

(a)

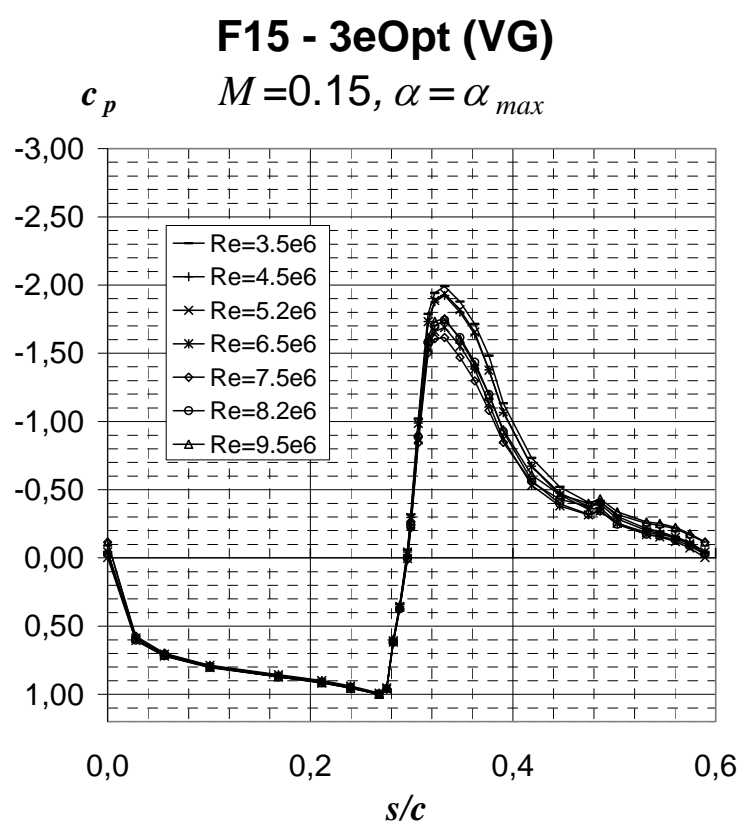

(b)

Figure 27.Pressure distributions at the flap for various Reynolds numbers at $M=0.15$ at the angle of attack for maximum lift coefficient of the configuration (a) F15-3eRef, and (b) F15-3eOpt configurations. 
F15 - VLCS

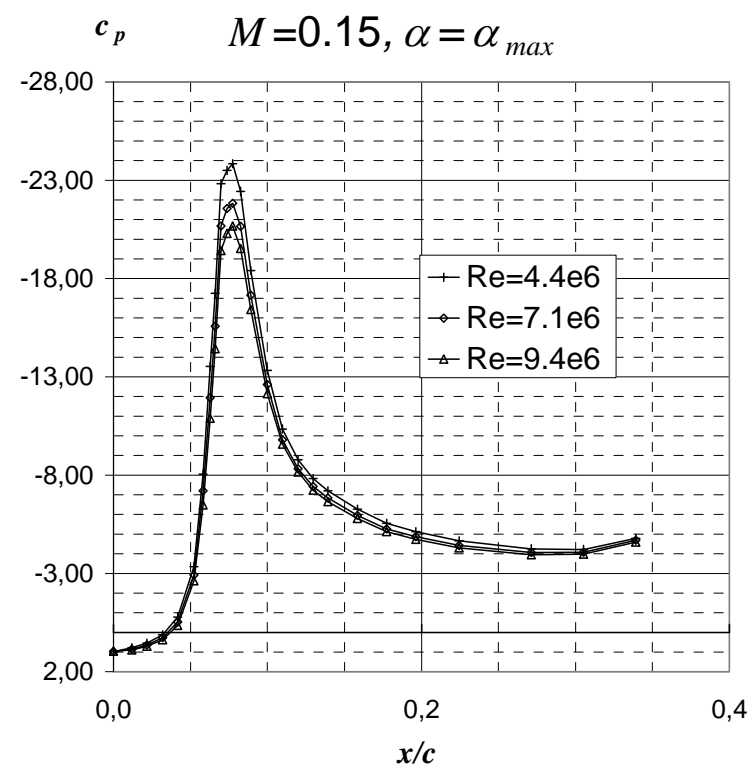

(a)

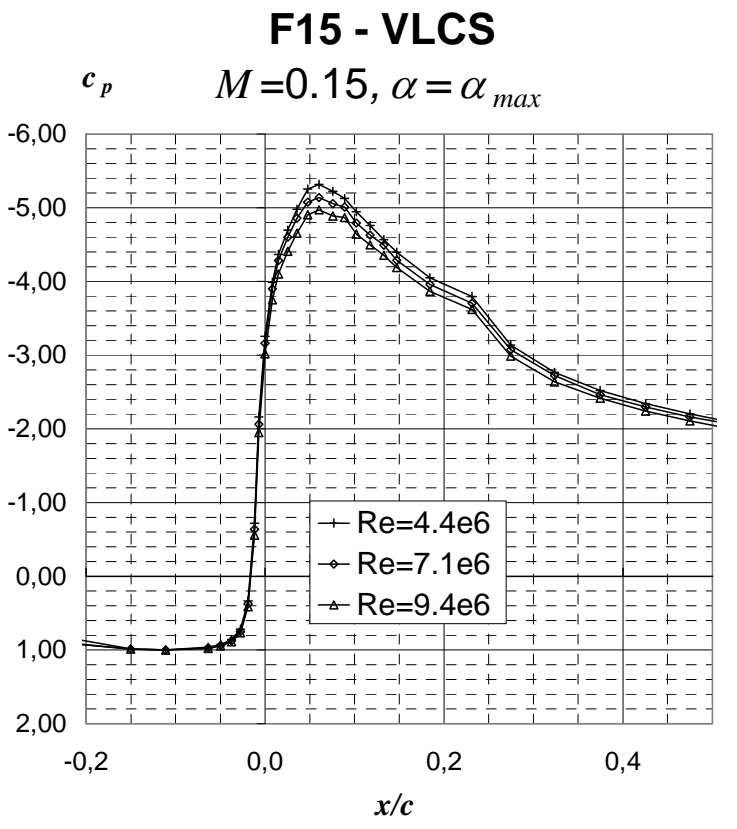

(b)

Figure 28.Pressure distributions along the local arc length of (a) the slat, and (b) the main wing of the F15VLCS configuration at $M=0.15$ for three Reynolds numbers in the range of decreasing maximum lift coefficient with increasing Reynolds number.

effects reducing the circulation is stronger than the ones for increasing the circulation. For the F15-VLCS configuration the conclusion is that the gap is optimum for a Reynolds number for $R e=4.5 \times 10^{6}$ and too large for higher Reynolds numbers. In fact the optimum slat gap seems to be very sensitive to the Reynolds number and setting design at a different Reynolds number may lead to non-optimum solutions.

Although not directly related to the stall behavior, the analysis also unveiled a similar sensitivity on the Reynolds number for the flap flow related to flap separation at low angles of attack. Figure 29 shows the evolution of the flap trailing edge pressure over the angle of attack for the investigated Reynolds numbers for all three configurations. The flap trailing edge pressure is a good indicator for flap separation. Attached flow is achieved if the trailing edge pressure coefficient is slightly positive, while negative values correspond to partial flap separation. The curves for all configurations at all Reynolds numbers show a tendency of pressure increase with increasing angle of attack in the lower range, thus signalizing the flap flow gets more stable the higher the angle of attack. After reaching a maximum the pressure drops again slightly. The reduction of the load on the flap is attributed to the increase of circulation on the main wing, which is directly reducing the flap suction by the "slat" effect and up to a configuration dependent angle of attack more significant than the change of the flow direction itself. By the induced velocity field of the wing the local flow direction onto the flap first decreases with increasing angle of attack. After reaching a most stable situation, the wing circulation increase doesn't completely compensate the increase of incidence leading to the slight flap trailing edge pressure drop. But there is more significant a very different behavior of the flap, which is of the same shape for all configurations but at different settings. The flap of the F15-3eRef configuration is attached at low Reynolds numbers, but separates significantly in the lower range of angles of attack at higher Reynolds numbers, but reattaches at about $\alpha=20^{\circ}$. The flap of the F15-3eOpt is attached over the complete range of incidences and Reynolds numbers, and gets only at the boundary of separation just before maximum lift. For the F15-VLCS the flap is separated at low Reynolds numbers for the complete range of angles of attack, and for higher Reynolds numbers it reattaches around $\alpha=10^{\circ}$, staying stable until the maximum lift coefficient is reached. Again this behavior is clearly attributed to the change of the effective gap which is influenced by the Reynolds number dependent boundary layer thicknesses at the flap and the lower wing trailing edge. The F15-3eRef flap is properly set for low Reynolds numbers. At higher Reynolds number the effective gap increases and the effective distance to the wing gets too large, so the reduction of the suction induced by the wing is not strong enough and the flap gets overloaded. For the F15-VLCS the gap is too small at low Reynolds numbers and the 
blockage of the gap flow results in a flap separation. The increase of effective gap here strengthens the circulation effect of the wing and the separation reduces for increasing Reynolds numbers. For the F15-3eOpt the tendency is the same as for the F15-VLCS in terms of a reduced flap loading with increased Reynolds number, but the flap is positioned at a less sensitive setting avoiding flap separation in the complete range of angels of attack up to shortly before stall. In conclusion - as for the slat - the optimum flap setting is highly dependent on the designated Reynolds number.

\section{F. Reynolds number dependencies of 2-element configurations}

Finally a look is taken towards the Reynolds number dependency of the maximum lift coefficient for the 2element configurations. Figure 30 shows this dependency for all three 2-element configurations. The F15-2eOpt test matrix has been measured in two different entries and in the figure only the data obtained in the second entry is shown. The F15-2eRef configuration shows a significant reduction of maximum lift with increasing Reynolds number, the configuration F15-2eOpt shows approximately no sensitivity on the Reynolds number, and the configuration F15-2eDN shows a Mach number dependent optimum. As already observed in all other configurations discussed before, measurements at constant temperature don't cover any of the effects in the same way.

In contrast to the 3-element configurations discussed above, the 2-element configurations give a better insight in the possible mechanisms since no additional effect of a slat is interfering. However, the look into the pressure distributions unveils partly similar effects on the flap as for the 3-element configurations reported above. Figure 31 shows pressure distributions at maximum lift coefficient in the region of the wing suction peak and the flap suction side for the F15-2eRef configuration for all Reynolds numbers at a Mach number $M=0.2$. Figure 32 displays the same for the F15-2eOpt configuration, and Figure 33 for the F15-2eDN configuration.

For the F15-2eRef, the suction at the main wing is not Reynolds number dependent, but the flap separation that is not existing at $R e=4.7 \times 10^{6}$ develops in size with increasing Reynolds number. The effective opening of the gap due to the reduction of boundary layer thicknesses reduces the effect of the wing on the flap. The flap seems to be at the limit of separation for the above mentioned Reynolds number, and the widening causes the flap to separate. This gets more severe as the effective gap is opening further.

The F15-2eOpt shows a slight decrease of the wing suction peak with increasing Reynolds number that is compensated by a similar slight increase of the flap suction. The compensation is nearly perfect in terms of achieved maximum lift coefficient making this configuration looking insensitive to the Reynolds number. In fact with increasing Reynolds number the lift center of the wing section is shifted towards the rear. The movement of the lift center can be attributed to a reduction of the "slat" effect of the wing onto the flap as the dominating mechanism.

The F15-2eDN shows more pronounced the opposite tendency as the F15-2eOpt, namely a rising suction level a wing and a decreasing suction beak on the flap. Interestingly, for this configuration the size of the flap separation is not depending on the Reynolds number. As already mentioned during the discussion of the Mach number effect, this configuration stalls at the flap first. By the increase of the Reynolds number this happens at a slightly higher angle of attack and the wing suction is accordingly increased. The therefore increased wing circulation dampens the flap suction. The balance of the shift is not as perfect as for the F15-2eOpt configuration. Therefore, the maximum of maximum lift coefficient over the Reynolds number is observed.

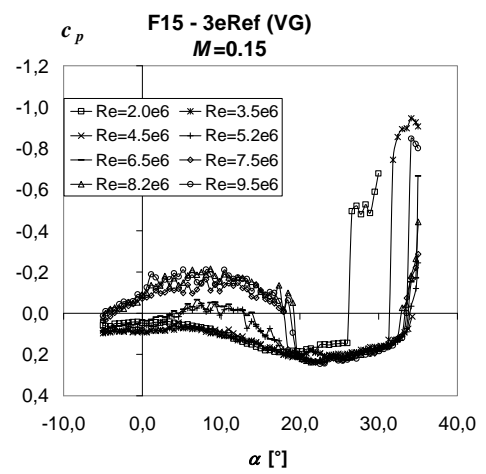

(a)

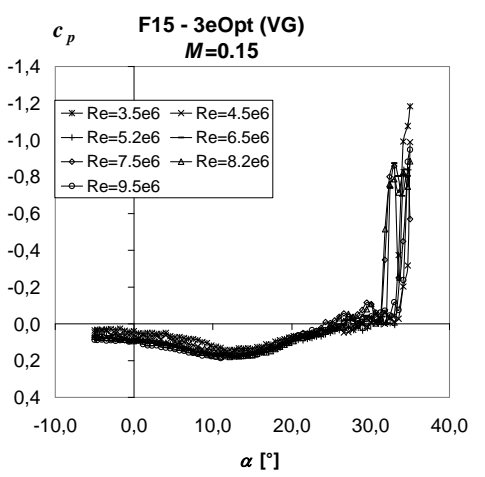

(b)

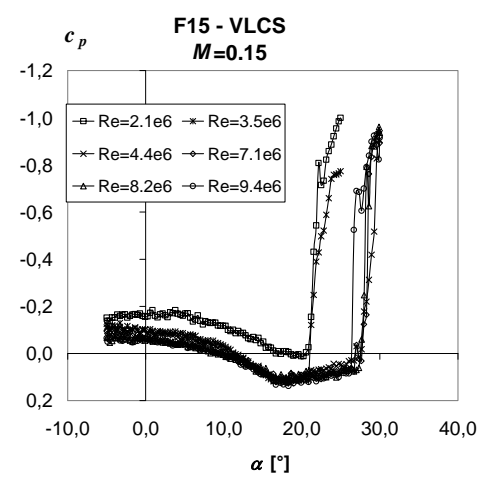

(c)

Figure 29.Evolution of the flap trailing edge pressure coefficient for different Reynolds numbers at $M=0.15$ for the configurations (a) F15-3eRef, (b) F15-3eOpt, and (c) F15-VLCS. 


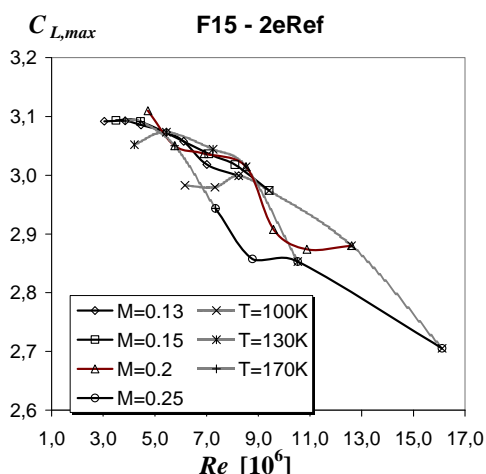

(a)

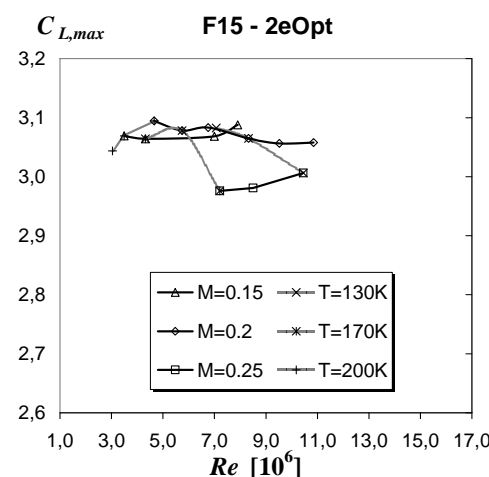

(b)

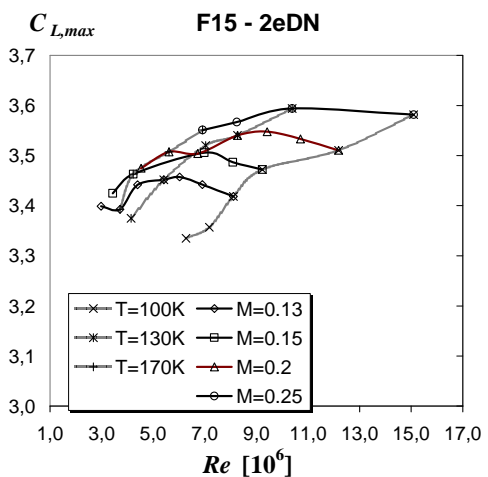

(c)

Figure 30.Dependency of maximum lift coefficient on the Reynolds number configurations at constant Mach number compared to dependency at constant temperature for the configurations (a) F15-2eRef, (b) F152eOpt, and (c) F15-2eDN.

\section{Conclusion}

A series of wind tunnel tests have been performed in the DNW-KKK cryogenic low speed facility with the DLR F15 two-dimensional high-lift model. The model has been equipped with different high-lift systems with and without slat device at different settings of the high-lift elements. The test conditions were chosen in order to assess the dependency of the stall characteristics on the Mach number and the Reynolds number independently. Details of the effects leading to differences in the stall behavior were shown by analyzing the pressure data in detail.

Although the Mach number range classically would be interpreted to be at the bound of incompressible flow, significant dependencies have been observed. Hereby, 3-element configurations are more sensitive to the Mach number than 2-element configurations. Local compressibility effects have been discovered even for the lowest Mach number used.

Stall mechanisms induced by supersonic regions dominate the limitations on the maximum lift coefficient for 3element airfoils. Nevertheless, it was shown that slats can operate up to the upper physical pressure limit related to $c_{p} M^{2}=-1$. The commonly assumed upper limit for the pressure rise at a slat element was discovered to not be a fixed number but is related mainly to the size of the slat element. For the 2-element configurations a maximum pressure rise that is depending on the flap setting is observed. Supersonic effects have only be observed for the highest Mach number $M=0.25$. In the case that supersonic regions appear the Reynolds number dependency gets less important.

For the 3-element configurations at lower Mach numbers a critical Reynolds number is observed below which the maximum lift coefficient is significantly reduced. It is assumed that this is related to the merge of the slat wake and the wing boundary layer, since this effect is not visible for 2-element configurations. In contrast to common assumptions, most of the configurations show a decreasing lift coefficient with increasing Reynolds number. Detailed analysis of the pressure distributions reveal that this is due to the change of the effective gap due to the change of the thicknesses of the boundary layers. On this basis all effects are explainable by basic multi-element airfoil theory.

Additionally, for the example of the 3-element configurations the well known effect of flap separations at low angles of attack, disappearing at higher angles of attack has been demonstrated together with the changing behavior related to the Reynolds number. In this context, but also for the Reynolds number sensitivities of the stall behavior itself of the investigated configurations, it has been also revealed that an optimum positioning of high-lift systems is only valid for a dedicated Reynolds number. This is of higher importance, since in the light of the presented results an appropriate assessment of high-lift performance cannot be made at a Reynolds number that is significantly different than for the real scale. Even for the highest Reynolds numbers shown here no asymptotic behavior of the maximum lift coefficient was observed. High Reynolds number testing is therefore a must for an accurate verification of the aerodynamic performance of high-lift configurations. 


\section{Acknowledgments}

The Author has to thank for the support of a number of colleagues involved in the setup and performance of the numerous wind tunnel campaigns. A special thank to Marvin Gülzow and Martin Sitzmann, DLR, and the crews of the DNW-KKK and DNW-NWB wind tunnels.

\section{References}

${ }^{1}$ Moir, I.R.M., "Measurements on a two-dimensional aerofoil with high-lift devices," No. A-2 in A Selection of Experimental Test Cases for the Validation of CFD Codes, AGARD AR-303, 1994.

${ }^{2}$ Brune, G.W., "Two-dimensional high-lift airfoil data for CFD code validation,” No. A-13 A Selection of Experimental Test Cases for the Validation of CFD Codes, AGARD AR-303, 1994.

${ }^{3}$ Valarezo,W. O., "High Lift Testing at High Reynolds Numbers,” AIAA Paper 92-3986, July 1992.

${ }^{4}$ Choudhari, M., and Visbal, M., "Second Workshop on Benchmark problems for Airframe Noise Computations", https://info.aiaa.org/tac/ASG/FDTC/DG/BECAN_files_BANCII.htm, accessed Dec. 2011.

${ }^{5}$ Dargel, G., Hansen, H., Wild, J., Streit, T., Rosemann, H., Richter, K., „Aerodynamische Flügelauslegung mit multifunktionalen Steuerflächen,“ DGLR, Bonn [Hrsg.]: DGLR Jahrbuch 2002, DGLR-2002-096, Vol. I, 2002, pp. 1605.

${ }^{6}$ Wild, J. Pott-Pollenske, M., and Nagel, B., ”An integrated design approach for low noise exposing high-lift devices,” AIAA Paper 2006-2843, 2006.

${ }^{7}$ Englar, R.J., "Development of pneumatic test techniques for subsonic high-lift and in-ground-effect wind tunnel investigations", Wall Interference, Support Interference and Flow Field Measurements, AGARD CP 535 (1993).

${ }^{8}$ Wild, J., Wichmann, G., Haucke, F., Peltzer, I., and Scholz P., ”Large scale separation flow control experiments within the German Flow Control Network,“ AIAA Paper 2009-0530, 2009.

${ }^{9}$ Mayer, J.P., "A Limit Pressure Coefficient and Estimation of Limit Forces on Airfoils at Supersonic Speeds,” NACA RM L8F23, 1948.

${ }^{10}$ Woodward, D.S., and Lean, D.E., “Where is High-Lift Today,” No. 1 in High-Lift System Aerodynamics, AGARD CP-515, 1993.

${ }^{11}$ Smith, A.M.O., “High-Lift Aerodynamics,” Journal of Aircraft, Vol. 12, No. 6, 1975, pp. 501-530. 


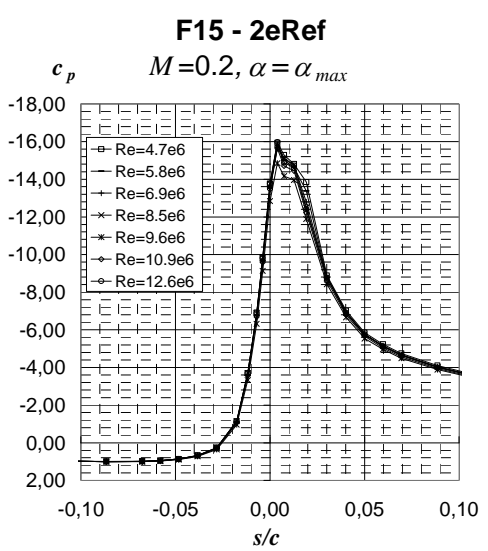

(a)

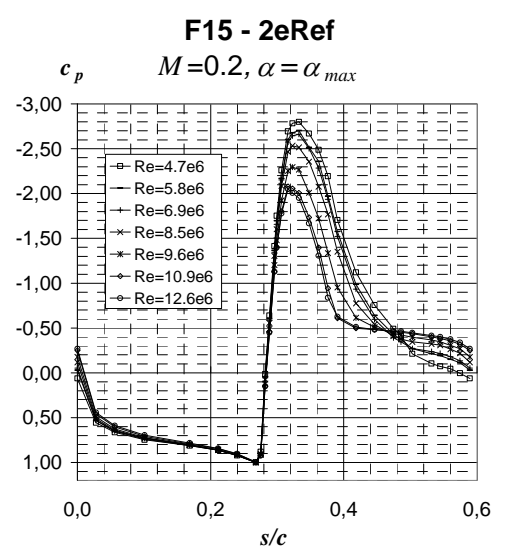

(b)

Figure 31.Pressure distributions for the F15-2eRef configuration for different Reynolds numbers at $M=0.2$ at (a) the suction peak of the main wing, and (b) at the flap.

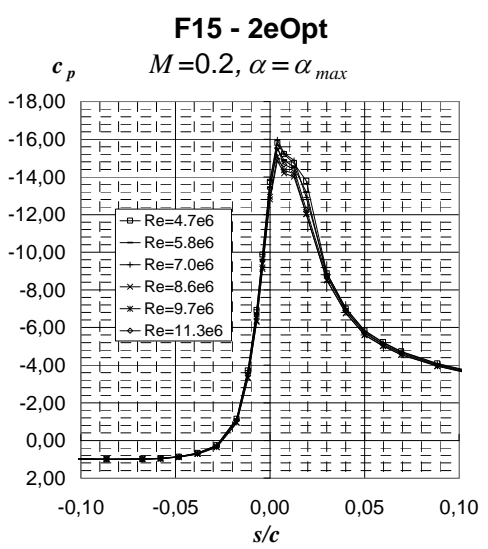

(a)

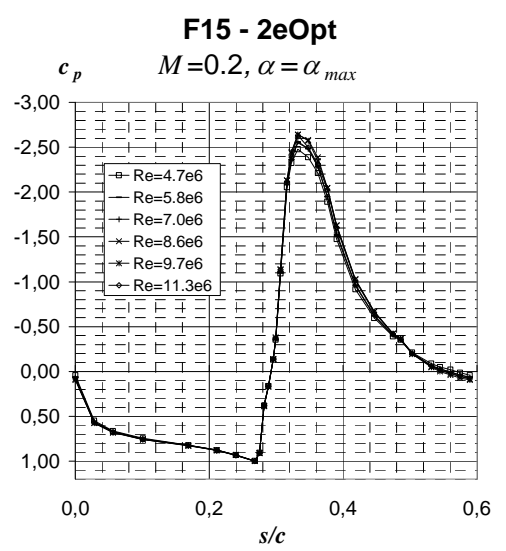

(b)

Figure 32.Pressure distributions for the F15-2eOpt configuration for different Reynolds numbers at $M=0.2$ at (a) the suction peak of the main wing, and (b) at the flap.

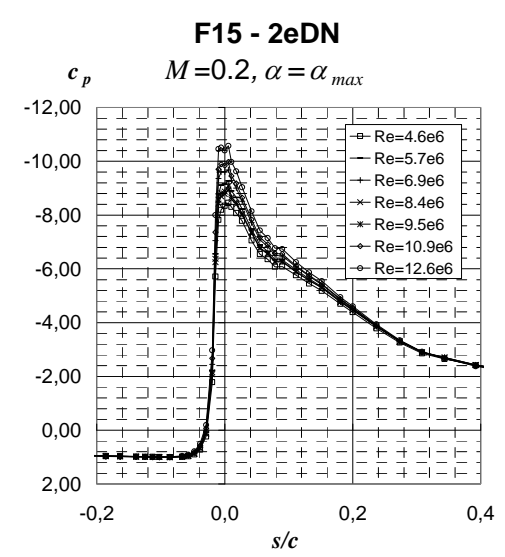

(a)

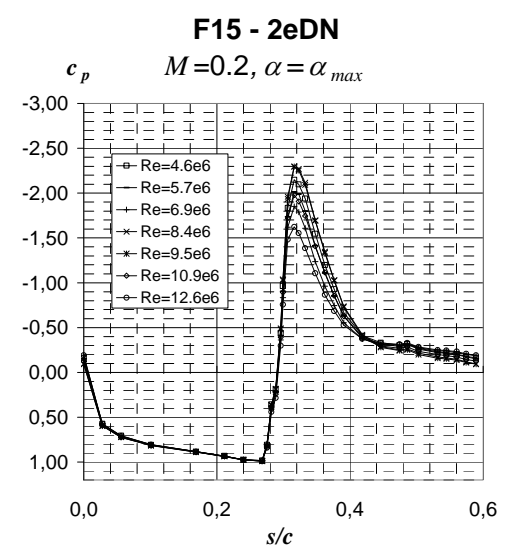

(b)

Figure 33.Pressure distributions for the F15-2eDN configuration for different Reynolds numbers at $M=0.2$ at (a) the suction peak of the main wing, and (b) at the flap. 